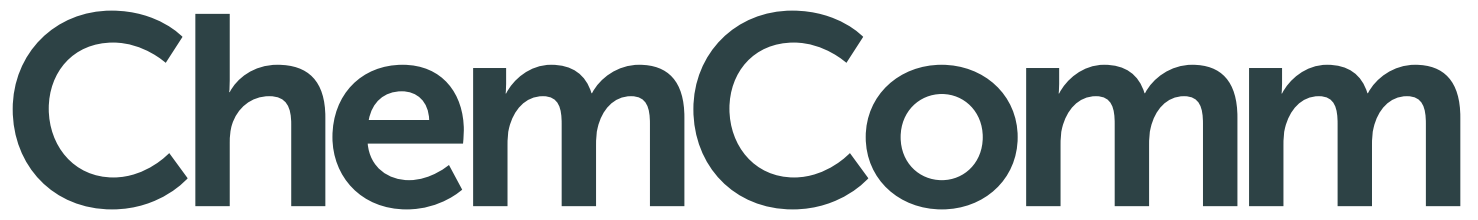

Chemical Communications

rsc.li/chemcomm

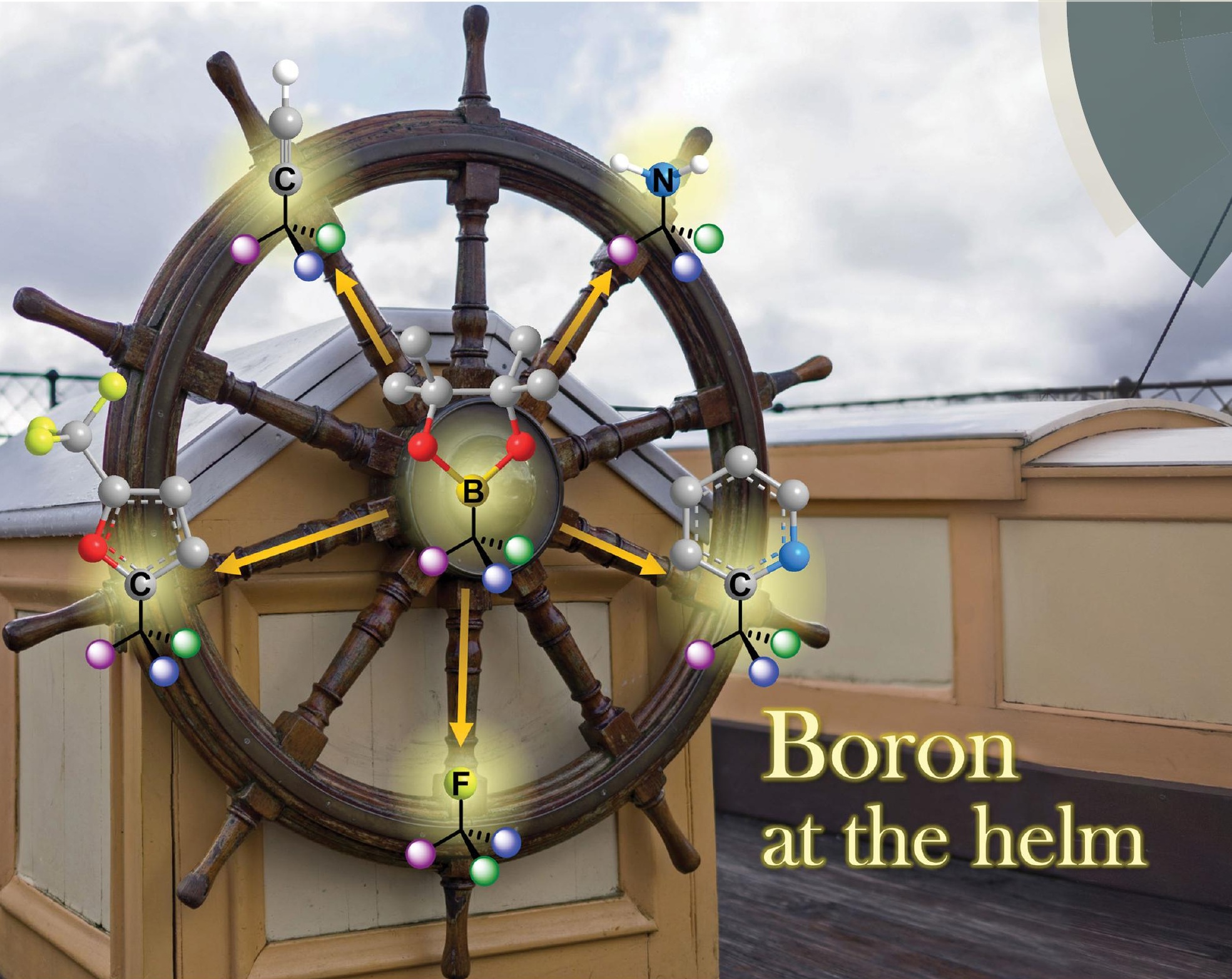

ISSN 1359-7345

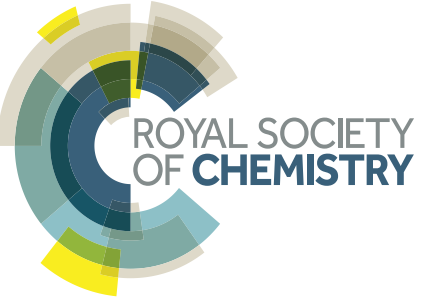




\section{D) Check for updates}

Cite this: Chem. Commun., 2017, 53,5481

Received 16th February 2017 Accepted 29th March 2017

DOI: $10.1039 / \mathrm{c} 7 \mathrm{cc} 01254 \mathrm{c}$

rsc.li/chemcomm

\title{
Stereospecific functionalizations and transformations of secondary and tertiary boronic esters
}

\author{
Christopher Sandford and Varinder K. Aggarwal (D) *
}

\begin{abstract}
The formation of highly enantioenriched boronic esters through both stoichiometric and catalytic methods has received much attention over the past decade. Accordingly, the transformations of the boronic ester moiety into other functional groups is of considerable interest in synthesis. Specifically, transformations which retain the high enantioenrichment of the starting boronic ester, either through a stereoretentive or a stereoinvertive pathway, lead to the formation of new $\mathrm{C}-\mathrm{C}, \mathrm{C}-\mathrm{O}, \mathrm{C}-\mathrm{N}, \mathrm{C}-\mathrm{X}$, or $\mathrm{C}-\mathrm{H}$ bonds at stereogenic centres. This feature article summarises the current state of the art in stereospecific transformations of both secondary and tertiary boronic esters into other functionalities and groups, whilst considering critically the transformations that are currently unattainable and would represent future advances to the field.
\end{abstract}

\section{Introduction}

Organoboron compounds are of significant utility in asymmetric synthesis. Accompanied with a large expansion in routes to obtain enantioenriched organoboron compounds, their subsequent transformations into a range of functional groups provide access to a broad array of diverse molecules with high enantioselectivity.

Asymmetric hydroboration, reported in 1961 by H. C. Brown, ${ }^{1}$ heralded the birth of modern asymmetric synthesis because, for the first time, it was shown that small molecules $\left(\mathrm{Ipc}_{2} \mathrm{BH}\right)$ were capable of providing high levels of enantioselectivity, levels that had previously been the sole preserve of enzymes. Stereospecific

School of Chemistry, University of Bristol, Cantock's Close, Bristol BS8 1TS, UK. E-mail:v.aggarwal@bristol.ac.uk oxidation of the chiral alkylboranes $\left(\mathrm{R}_{3} \mathrm{~B}\right)$ formed through hydroboration gave enantioenriched alcohols. Subsequently, further stereospecific transformations of the alkylboranes were elucidated, enabling the $\mathrm{C}-\mathrm{B}$ bond to be converted into a wide range of other functional groups. ${ }^{2}$

However, despite the range of reactions that can be carried out using alkylboranes, their synthetic utility is tempered by their air and moisture sensitivity. Changing the organoborane into a boronic ester $\left(\mathrm{RB}\left[\mathrm{OR}^{\prime}\right]_{2}\right)$ enables easier purification, especially in the case of boronic acid pinacol esters (RBpin). ${ }^{3}$ This characteristic facilitates the use of boronic esters in synthesis and as a tool to create new bonds. Perhaps most notably, the Suzuki-Miyaura reaction has been applied extensively because of operational simplicity and the range of arylboronic esters and acids that are commercially available. ${ }^{4}$

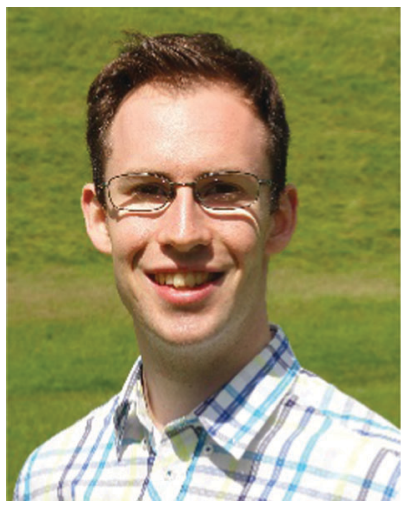

Christopher Sandford was born in Bristol, UK in 1990. He obtained his MChem from the University of Oxford in 2013, conducting research under the supervision of Prof. Darren Dixon. Chris is currently pursuing his $\mathrm{PhD}$ at the University of Bristol under the direction of Prof. Varinder Aggarwal, where he is investigating the reactivity of boronate complexes.
Christopher Sandford

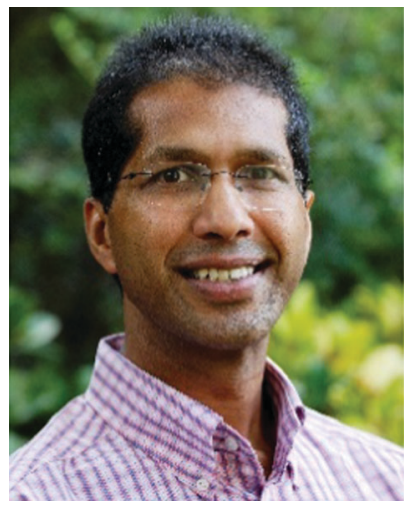

Varinder K. Aggarwal
Varinder K. Aggarwal studied chemistry at Cambridge University and received his $P h D$ in 1986 under the guidance of Dr Stuart Warren. After postdoctoral studies (1986-1988) under Prof. Gilbert Stork, Columbia University, he returned to the UK as a Lecturer at Bath University. In 1991 he moved to Sheffield University, where he was promoted to Professor in 1997. In 2000 he moved to Bristol University where he holds the Chair in Synthetic Chemistry. He was elected Fellow of the Royal Society in 2012. 
As a tool for chiral synthesis, the development of novel methods to obtain enantioenriched alkylboronic esters has received much attention over the past decade. Methods to introduce the boronic ester moiety into organic compounds with high levels of asymmetric induction include both stoichiometric methods (such as homologation $^{5}$ and lithiation-borylation ${ }^{6}$ ) and catalytic methods (such as hydroboration, ${ }^{7}$ conjugate addition ${ }^{8}$ and diboration ${ }^{9}$ ). Having established routes to the synthesis of these molecules, attention has also turned to their manipulation and subsequent stereospecific transformations into a wide range of functional groups, ${ }^{10}$ in a similar vein to the range of transformations open to alkylboranes.

In this feature article, we review the range of stereospecific transformations that are currently available to secondary and tertiary boronic esters, operating through either a stereoretentive or a stereoinvertive pathway (i.e. not a radical pathway). The transformations covered (Fig. 1) include oxidations ( $\mathrm{C}-\mathrm{O}$ bond formation), conversions of boron to other heteroatoms (C-N, $\mathrm{C}-\mathrm{X}$ ), protodeboronation $(\mathrm{C}-\mathrm{H})$, as well as the formation of new $\mathrm{C}-\mathrm{C}$ bonds (resulting from homologations, olefinations, alkynylations and coupling reactions). These transformations have been selected for their applicability to any general alkylboronic ester, and so reactions that are dependent on the presence of specific functional groups (such as allylic boronic esters, or coupling reactions activated by neighbouring directing groups), except for benzylic boronic esters, have been omitted. With this review, we aim to showcase the work that we, and others, have conducted to expand the synthetic utility of enantioenriched boronic esters, whilst also identifying transformations that remain elusive and would represent significant future advances to the field.

\section{Carbon-heteroatom bond formation}

\subsection{Oxidation of boronic esters to alcohols}

The stereospecific oxidation of boronic esters to the corresponding alcohol is the most versatile and widely used functionalization reaction of boronic esters. Building upon the oxidation of organoboranes through the use of basic hydrogen peroxide, developed by Brown and co-workers in $1961,{ }^{11}$ the reaction of an enantioenriched boronic ester under these conditions affords the alcohol with complete retention of configuration. Addition of the peroxide anion to the empty p-orbital of the boron atom forms boronate complex 1 (Scheme 1). The boronate complex then undergoes a 1,2-metallate rearrangement - migration of the $\mathrm{C}-\mathrm{B} \sigma$-bond onto the adjacent oxygen atom, with loss of hydroxide. The nature of this 1,2-metallate rearrangement step is such that the stereoconfiguration of the carbon initially attached to boron is retained, a feature that is common to many of the stereospecific transformations discussed throughout this review. Finally, hydrolysis cleaves the $\mathrm{O}-\mathrm{B}$ bond to afford alcohol 3. The oxidation of a boronic ester is slower than for analogous boranes owing to donation of the oxygen lone pairs into the empty p-orbital of boron, an attribute that decreases the electrophilicity of the boron.

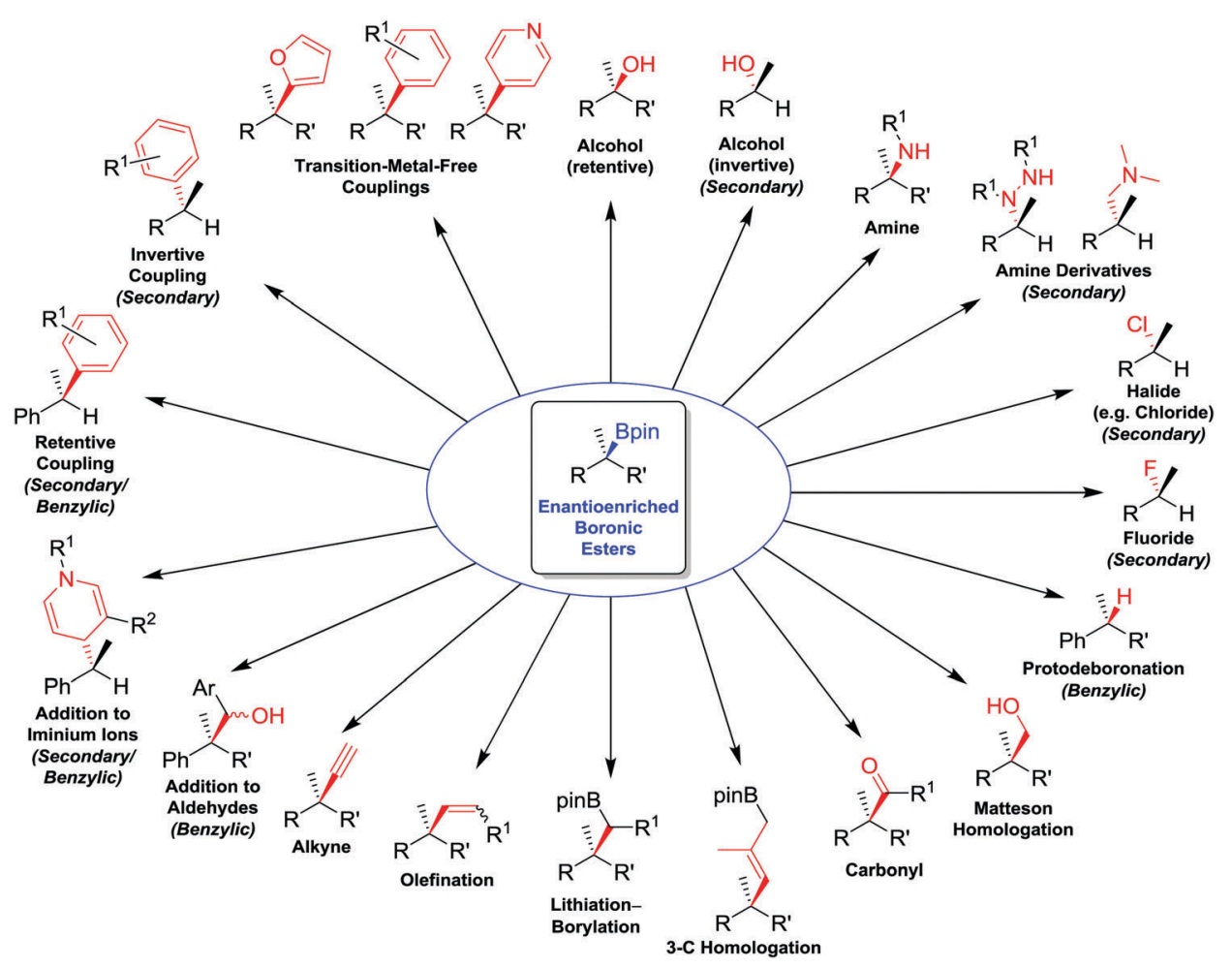

Fig. 1 Summary of stereospecific functional group transformations of secondary and tertiary boronic esters. 


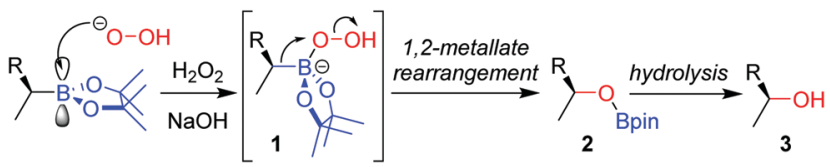

Scheme 1 Mechanism of the oxidation of boronic esters by basic hydrogen peroxide.

Although numerous synthetic routes are available to produce primary and secondary alcohols, the formation of stereodefined tertiary alcohols is significantly less developed. We found that oxidation can also be applied to tertiary boronic esters (Scheme 2), ${ }^{12}$ which can be prepared by lithiation-borylation (see Section 4.3). During the course of our studies on the oxidation of tertiary boronic esters, we have found that it is sometimes advantageous to add dibutylhydroxytoluene (BHT) to prevent the formation of peroxy radicals, and ethylenediaminetetraacetic acid (EDTA) to minimize the decomposition of hydrogen peroxide by trace metals. ${ }^{12,13}$ Furthermore, we find that THF is an ideal solvent for improving miscibility with the aqueous phase.

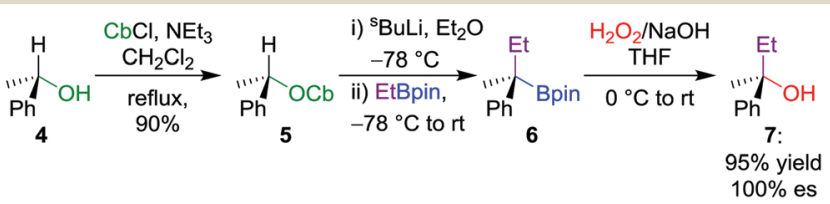

Scheme 2 Conversion of chiral secondary alcohols into tertiary alcohols by lithiation-borylation (see Section 4.3) and the stereospecific oxidation of boronic esters. $\mathrm{OCb}=\mathrm{N}, \mathrm{N}$-diisopropylcarbamate.

As a milder alternative to the hydrogen peroxide conditions, sodium perborate effects the oxidation with higher functionalgroup tolerance.$^{14}$ For example, Fontani et al. reported that the oxidation of diastereomerically pure cyclopropylboronic acid pinacol ester $\mathbf{8}$ with hydrogen peroxide led to a poor conversion along with the formation of ring-opened carbonyl compounds. ${ }^{15}$ Conversely, the oxidation with sodium perborate was found to afford the desired alcohol 9 in $81 \%$ yield (Scheme 3). Furthermore, sodium perborate is also often the reagent of choice for oxidations where the boron moiety is situated $\beta$ to an electron-withdrawing group (such as a sulfone or a carbonyl group). ${ }^{16}$ Household bleach (aq. NaOCl) has also been used to oxidise a tertiary $\beta$-keto boronic ester with complete retention of configuration. ${ }^{17}$

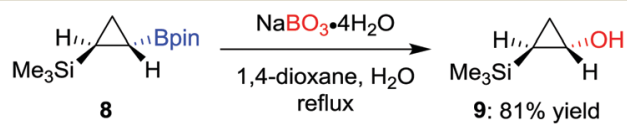

Scheme 3 Oxidation of cyclopropylboronic esters using sodium perborate by Fontani et al.

\subsection{Amination of boronic esters}

Although trialkylboranes can be readily converted into the corresponding amines by reaction with reagents such as chloramine ${ }^{18}$ or alkyl azides, ${ }^{19}$ the reduced Lewis acidity of boronic esters prevents reactivity with these weakly Lewis basic

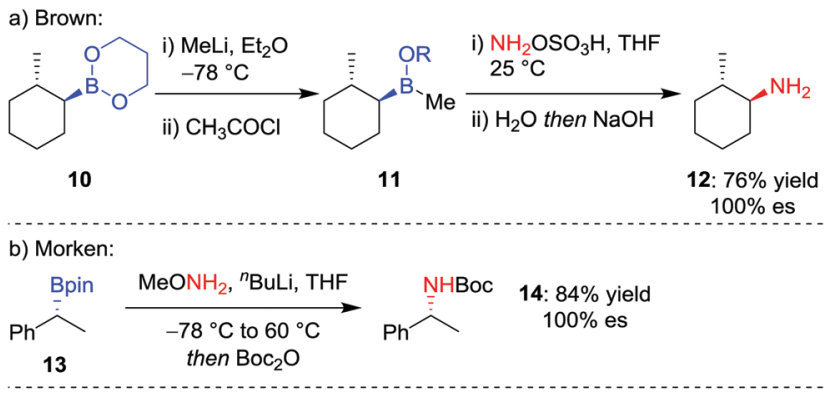

c) Aggarwal:

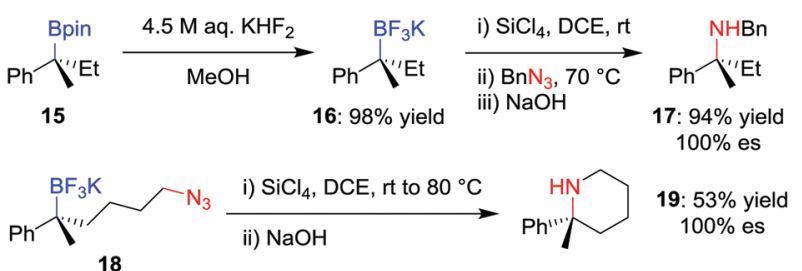

Scheme 4 Procedures for the stereospecific amination of secondary and tertiary boronic esters. $\mathrm{R}=\mathrm{CH}_{2}\left(\mathrm{CH}_{2}\right)_{2} \mathrm{OC}(\mathrm{O}) \mathrm{CH}_{3}$.

reagents due to ineffective association. To facilitate the stereospecific amination of boronic acid glycol esters 10, Brown and co-workers (Scheme 4a) reported the initial conversion into the more Lewis acidic borinic ester $\mathbf{1 1}$ with methyllithium and subsequent amination by using hydroxylamine-O-sulfonic acid $\left(\mathrm{NH}_{2} \mathrm{OSO}_{3} \mathrm{H}\right) \cdot{ }^{20}$

Seeking a direct stereospecific amination protocol for alkylboronic acid pinacol esters, Morken and co-workers ${ }^{21}$ (Scheme $4 \mathrm{~b}$ ) proposed increasing the nucleophilicity of the amination reagent by using lithiated alkoxy amines. Reaction of enantioenriched boronic ester 13 with 3.0 equivalents of methoxyamine and $n$-butyllithium, followed by Boc protection, was found to afford Boc-protected amine 14 in $84 \%$ yield. This transformation was found to proceed with complete retention of stereoconfiguration for secondary boronic esters, an outcome that is consistent with a 1,2-metallate rearrangement involving migration of the $\mathrm{C}-\mathrm{B} \sigma$-bond onto the nitrogen atom with loss of methoxide. However, these conditions were not amenable for the amination of tertiary alkylboronic esters.

Although a method for the direct amination of tertiary boronic esters has yet to be reported, we sought to provide the desired tertiary amines by first converting the boronic ester $\mathbf{1 5}$ into the trifluoroborate salt 16 (Scheme 4c), ${ }^{22}$ which can be achieved in essentially quantitative yields using $\mathrm{KHF}_{2}$ in $\mathrm{MeOH}^{23}$ Pleasingly, we found that a modification of Matteson's amination conditions for trifluoroborate salts, ${ }^{24}$ using $\mathrm{SiCl}_{4}$ to form the alkyldichloroborane ${ }^{25}$ and then reaction with an alkylazide, ${ }^{19 b}$ led to the corresponding amine 17 through a retentive pathway without loss in stereochemical integrity. The reaction can occur intramolecularly to obtain piperidines (19), and we subsequently discovered that the methodology can also be applied to non-benzylic tertiary boronic esters. $^{26}$

\subsection{Boronate complexes as chiral organometallic-type reagents}

In addition to the functionalization of boronic esters via the stereospecific migration of the $\mathrm{C}-\mathrm{B}$ bond, we envisaged that 
enantioenriched boronic esters could serve as precursors to chiral organometallic-type nucleophilic reagents. ${ }^{27}$ We proposed that the boronic ester could be activated by addition of an electron-rich aryllithium to form a nucleophilic boronate complex. The complex is configurationally stable and should thus confer the chirality of the starting material into the desired functionalized compound.

Upon addition of $p$ - $\mathrm{MeOC}_{6} \mathrm{H}_{4} \mathrm{Li} 21$ to boronic ester 20 (Scheme $5 \mathrm{a}),{ }^{28}$ followed by reaction with $\mathrm{I}_{2}$ as the electrophile, the desired C-I bond was formed in $80 \%$ yield and $97 \%$ es. It was determined that the reaction is invertive at the stereogenic carbon (a polar, $\mathrm{S}_{\mathrm{E}} 2 \mathrm{inv}$ pathway). However, high enantiospecificity was not observed when using diisopropyl azodicarboxylate (DIAD) as the electrophile: hydrazine 25 was formed in $82 \%$ yield and only $13 \%$ es (Scheme $5 \mathrm{~b}$ ). We postulated that the low enantiospecificity resulted from a competing single-electron transfer (SET) pathway (Fig. 2), a mechanism that is supported by radical-clock experiments. Nonetheless, the SET pathway could be reduced by adjusting the electronics of the aryllithium used to form the boronate complex; by using the electron-deficient aryllithium $\mathbf{2 4}$, the enantiospecificity of the reaction was found to increase to $66 \%$.

With the optimal nucleophilic boronate complex in hand, we were able to functionalize the $\mathrm{C}-\mathrm{B}$ bond of enantioenriched secondary boronic esters with a range of different heteroatom electrophiles with up to complete stereoinversion - enabling
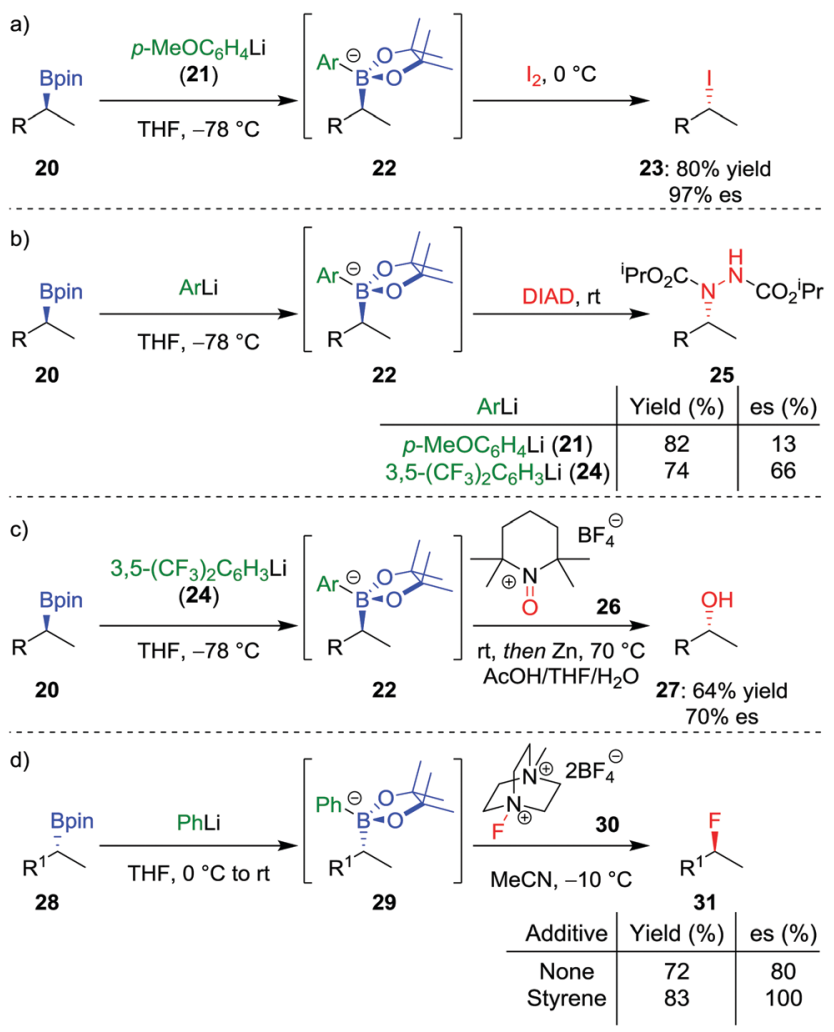

Scheme 5 Stereospecific conversions of secondary boronic esters into other heteroatoms by the reactions of nucleophilic boronate complexes with electrophiles. $\mathrm{R}=\mathrm{CH}_{2} \mathrm{CH}_{2} \mathrm{Ph}, \mathrm{R}^{1}=\mathrm{CH}_{2} \mathrm{CH}_{2} \mathrm{C}_{6} \mathrm{H}_{4} \mathrm{OMe}, \mathrm{DIAD}=$ ${ }^{i} \mathrm{PrO}_{2} \mathrm{C}-\mathrm{N}=\mathrm{N}-\mathrm{CO}_{2}{ }^{\mathrm{i}} \mathrm{Pr}$.

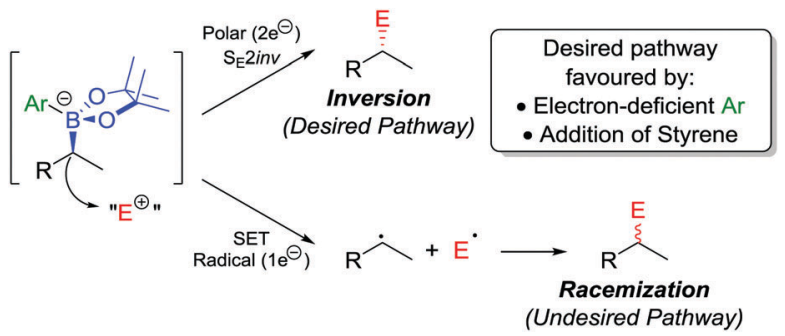

Fig. 2 Reaction pathways of chiral boronate complexes. $\mathrm{E}^{+}=$electrophile, SET = single-electron transfer.

iodination, chlorination, bromination, as well as the formation of $\mathrm{C}-\mathrm{N}$ bonds. $\mathrm{C}-\mathrm{O}$ bonds can also be formed using this stereoinvertive method, thus being complementary to the standard oxidation conditions which are stereoretentive (see Section 2.1). For example, reaction of the boronate complex with 2,2,6,6tetramethylpiperidine-1-oxoammonium tetrafluoroborate (26) and subsequent $\mathrm{N}-\mathrm{O}$ bond cleavage affords the corresponding alcohol 27 in $64 \%$ yield and $70 \%$ es (Scheme $5 \mathrm{c}$ ). The reaction was found to be applicable to both benzylic and non-benzylic substrates, with the former exhibiting both enhanced reactivity and enantiospecificity. These reactions are related to the halogenation of boronate complexes derived from chiral boranes which also occurred with inversion. ${ }^{29}$

The range of heteroatoms that can be introduced using a boronate complex was further expanded to include fluorine, ${ }^{30}$ providing access to enantioenriched alkylfluorides (Scheme 5d). Boronate complex 29 was formed by reacting boronic ester 28 with phenyllithium, and was then reacted with Selectfluor II (30) in acetonitrile, resulting in the formation of alkylfluoride $\mathbf{3 1}$ in $72 \%$ yield, but only $80 \%$ es. We discovered that the addition of styrene as a cheap and readily-available additive enhanced the enantiospecificity, enabling the product to be formed in $83 \%$ yield and complete enantiospecificity. Interestingly, the use of styrene led to no measurable decrease in yield in the reaction, suggesting that it was not merely acting as a radical trap for the SET pathway. Instead, we proposed that a fast radical propagation mechanism is inhibited by styrene, which acts as a radical scavenger (Fig. 3), thus allowing the desired $\mathrm{S}_{\mathrm{E}}$ 2inv pathway to dominate.

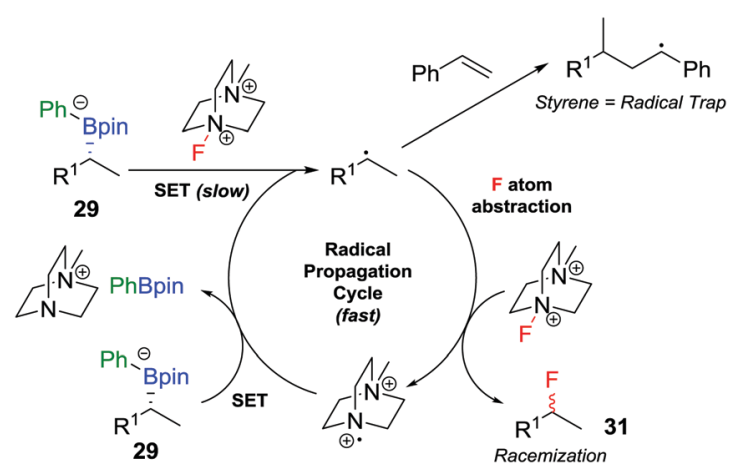

Fig. 3 Undesired radical pathways for the fluorination of boronate complexes, leading to the formation of racemic product, unless prevented by styrene. 
In order to determine the range of electrophiles that might be suitable for reactions with boronate complexes, we sought to determine the nucleophilicity parameter $(N)$ of the complexes to compare with other nucleophiles. ${ }^{31}$ In collaboration with Mayr and co-workers, we discovered that the aryl group has a significant effect on reactivity: the boronate complex derived from phenyllithium is 100 times more reactive than that derived from 3,5- $\left(\mathrm{CF}_{3}\right)_{2} \mathrm{C}_{6} \mathrm{H}_{3} \mathrm{Li}$ (24). ${ }^{32}$ Furthermore, the order of the nucleophilicity parameter follows the trend: secondary benzylic $>$ primary alkyl $>$ primary benzylic $>$ secondary alkyl. With these relative levels of reactivity known, we aim to explore other electrophiles that should participate in this $\mathrm{S}_{\mathrm{E}} 2 \mathrm{inv}$ pathway (see also Section 4.5).

\section{Carbon-hydrogen bond formation}

\subsection{Protodeboronation of tertiary benzylic boronic esters}

The stereospecific conversion of the $\mathrm{C}-\mathrm{B}$ bond of a tertiary boronic ester into a $\mathrm{C}-\mathrm{H}$ bond represents an efficient synthesis of tertiary alkyl stereogenic centres, including 1,1-diarylalkanes. Tertiary boranes are known to undergo protodeboronation by using propionic acid as the reagent of choice, ${ }^{33}$ owing to strong $\mathrm{B}-\mathrm{O}$ bond formation driving the reaction. We discovered that this reagent is not efficient for the protodeboronation of tertiary benzylic boronic esters, ${ }^{34}$ leading to slow conversion predominantly towards elimination products. We reasoned that the reaction could be facilitated if the driving force was changed to the formation of stronger B-F bonds. Consequently, we found that the addition of CsF and $\mathrm{H}_{2} \mathrm{O}$ in dioxane at $30{ }^{\circ} \mathrm{C}$ leads to the protodeboronation of diarylalkyl boronic ester 32 in 91\% yield and 99\% es (Scheme 6a), via a stereoretentive pathway. The methodology also provides access to enantioenriched deuterated tertiary alkanes by simply substituting the $\mathrm{H}_{2} \mathrm{O}$ with $\mathrm{D}_{2} \mathrm{O}$.

In contrast with diarylalkyl boronic esters, aryldialkyl boronic esters were found to be significantly more difficult to protodeboronate. Nevertheless, using the more reactive $\mathrm{TBAF} \cdot 3 \mathrm{H}_{2} \mathrm{O}$ enabled the protodeboronation of 35 in 99\% yield and 99\% es when conducted in $n$-pentane (Scheme $6 \mathrm{~b}$ ). Notably, the protodeboronation conditions require activated substrates, such as a benzylic (or allylic ${ }^{35}$ ) boronic ester. However, our research group found that non-benzylic boronic esters could undergo protodeboronation by employing oxidative conditions, leading to

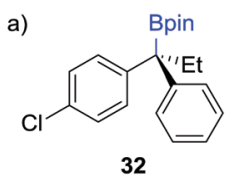

32

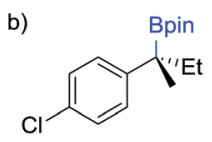

35

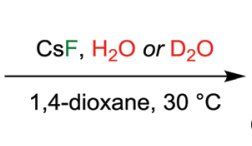

$\mathrm{R}=\mathrm{H}, \mathbf{3 3}: \mathbf{9 1 \%}$ yield, $99 \%$ es $\mathrm{R}=\mathrm{D}, \mathbf{3 4}: \mathbf{9 7 \%}$ yield, $96 \%$ es

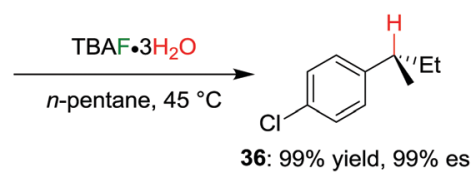

Scheme 6 Protodeboronation of tertiary dibenzylic and monobenzylic boronic esters.

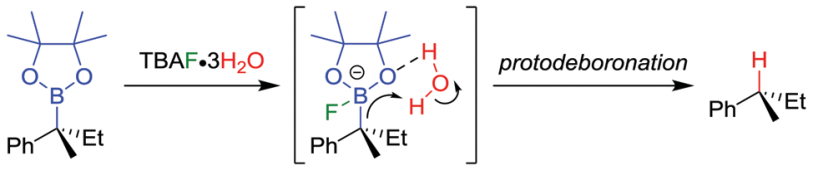

Scheme 7 Proposed mechanism for the stereoretentive protodeboronation of tertiary benzylic boronic esters.

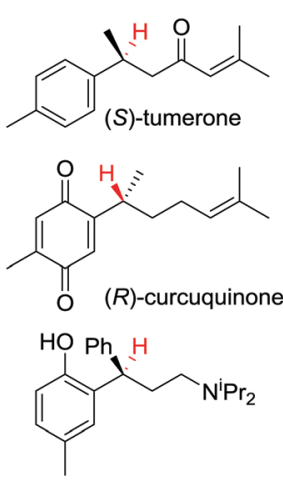

(R)-tolterodine
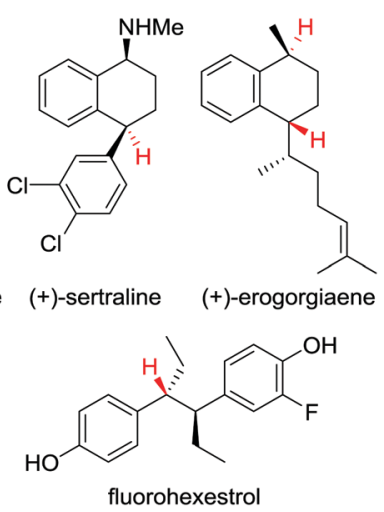

Fig. 4 Examples of biologically and therapeutically active molecules synthesised using a protodeboronation step to introduce the displayed hydrogen(s) enantiospecifically.

homolytic cleavage of the $\mathrm{C}-\mathrm{B}$ bond, ${ }^{36}$ the resulting $\mathrm{C}-\mathrm{H}$ bond being formed without stereospecificity owing to the radical nature of the intermediates.

The TBAF $3 \mathrm{H}_{2} \mathrm{O}$ reaction is believed to occur via a hydrogenbonded boronate complex, which delivers the proton to the same side as the boron atom (Scheme 7). In more polar solvents, solvation of the water molecules is proposed to cause a competing invertive pathway and subsequent erosion in enantiospecificity.

This protodeboronation strategy provides ready access to enantioenriched alkanes that are otherwise difficult to access, since the newly formed stereogenic centre can be placed distal to other functional groups. Indeed, we have applied the methodology to the synthesis of a wide range of biologically and therapeutically active molecules (Fig. 4). ${ }^{34,37}$

\section{Carbon-carbon bond formation}

\subsection{One-carbon homologations}

The homologation of an enantioenriched boronic ester ${ }^{38}$ by a one-carbon unit allows facile chain extension whilst retaining the valuable boronic ester group for future functionalization. The simplest such homologation is the insertion of a methylene $\left(\mathrm{CH}_{2}\right)$ unit into the $\mathrm{C}-\mathrm{B}$ bond, a reaction that was initially reported by Sadhu and Matteson in $1985 .{ }^{39}$ In their seminal work, it was found that addition of $n$-butyllithium to a mixture of boronic ester 37 and iodochloromethane in THF at $-78{ }^{\circ} \mathrm{C}$ affords the homologated boronic ester 39 in $95 \%$ yield, a transformation that proceeds through a stereoretentive 1,2-metallate rearrangement (Scheme 8a). Initially, iodine-lithium exchange forms $\mathrm{LiCH}_{2} \mathrm{Cl}$, which adds to the empty boron p-orbital to form boronate complex 38, thus triggering the 1,2-metallate rearrangement with 


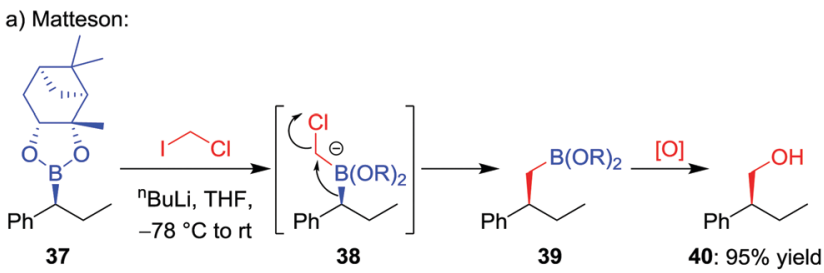
$37 \quad 38 \quad 30.95 \%$ yield b) Aggarwal: 41: $100 \%$ es

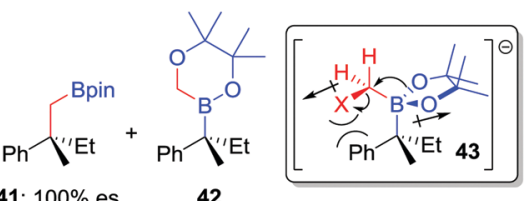

\begin{tabular}{c|c|c|c} 
Reagent & $\mathbf{X}$ & $\mathbf{4 1}(\%)$ & $\mathbf{4 2}(\%)$ \\
\hline $\mathrm{LiCH}_{2} \mathrm{Cl}$ & $\mathrm{Cl}$ & 71 & 20 \\
$\mathrm{LiCH}_{2} \mathrm{Br}$ & $\mathrm{Br}$ & 83 & 5
\end{tabular}
yield of $\mathbf{4 2}$ determined by ${ }^{11} \mathrm{~B}$ NMR analysis of the crude material. homologation procedure. stereoretention.
Scheme 8 Stereospecific Matteson homologations of enantioenriched boronic esters. Yield and enantiospecificity of $\mathbf{4 1}$ recorded after oxidation;

loss of chloride. A number of alternative protocols were subsequently investigated by the groups of Matteson and Brown, ${ }^{40}$ including in situ reduction of $\alpha$-chloroboronic esters, ${ }^{41}$ with bromochloromethane becoming the reagent of choice for the Matteson

When we investigated the homologation of tertiary boronic esters, ${ }^{26,42}$ we discovered that the use of bromochloromethane led to competitive oxygen migration, forming borinic ester $\mathbf{4 2}$ (Scheme 8b). However, through analysis of conformation 43, we reasoned that using bromide as a bulkier and less-polar leaving group would disfavour oxygen migration; indeed, when using in situ formed bromomethyllithium, the carbonmigration product 41, which was subsequently oxidized to the alcohol, was formed in $83 \%$ yield and with complete

Computational studies on the effect of sterics in this migration conducted by Elliott et al. have shown that the energy difference between the barriers to carbon- and oxygenmigrations are significantly reduced for sterically bulky tertiary boronic esters, and that decomplexation and/or undesired reactions of the boronate complex can take place in these cases. ${ }^{43}$ Consequently, greater yields were found by decreasing steric hindrance around the boron atom through replacing the pinacol ligand with propane-1,3-diol.

Manipulation of the oxidation state of the carbon atom introduced from the organolithium allows access to alternative homologated functional groups. Using $\mathrm{LiCHCl}_{2}$ (derived from the deprotonation of $\mathrm{CH}_{2} \mathrm{Cl}_{2}$ ) provides access to the homologated aldehyde $\mathbf{4 5}^{\mathbf{4 4}}$ after oxidation of the intermediate $\alpha$-chloroboronic ester 44 (Scheme $9 a){ }^{45}$ For a tertiary boronic ester, we have found the homologation reaction to proceed in $68 \%$ yield with complete stereoretention (Scheme $9 \mathrm{~b}) .{ }^{42}$ Fandrick et al. have subsequently applied this homologation on a decagram scale to install a quaternary stereogenic centre in their synthesis of 5-lipoxygenase-activating protein (FLAP) inhibitor $\mathbf{5 0}$ (Scheme 9c). ${ }^{46}$
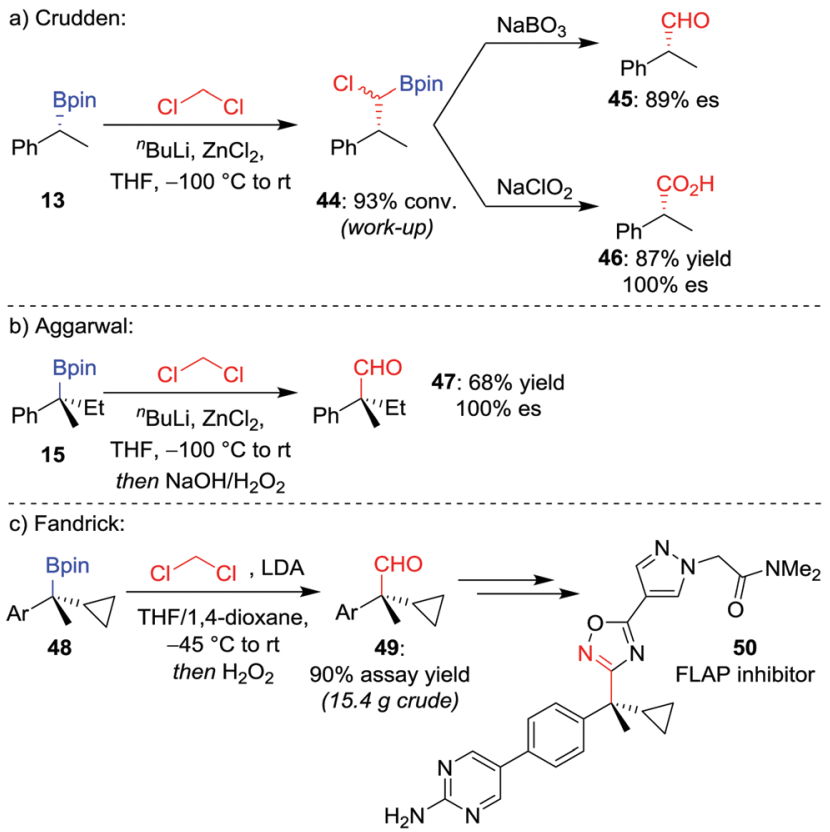

Scheme 9 One-carbon homologations of boronic esters to aldehydes and carboxylic acids. $\mathrm{Ar}=p-\mathrm{BrC}_{6} \mathrm{H}_{4}$

For the homologation to the corresponding carboxylic acid, Crudden and co-workers found the direct homologation to be challenging, ${ }^{44}$ but can be achieved via a two-step procedure involving oxidation of the $\alpha$-chloroboronic ester 44 with $\mathrm{NaClO}_{2}$ (Scheme 9a). This two-step strategy had previously been applied by Matteson and Beedle to oxidise $\alpha$-azido boronic esters into the corresponding carboxylic acids in their synthesis of enantioenriched $\alpha$-amino acids. ${ }^{47}$ As an alternative route, we have also applied a two-step procedure involving oxidation of the homologated alcohol with pyridinium dichromate. ${ }^{48}$

\subsection{Three-carbon homologations}

As an alternative to one-carbon homologation processes where the 1,2-metallate rearrangement proceeds by an $\mathrm{S}_{\mathrm{N}} 2$-type displacement of a leaving group (see Section 4.1), an $\mathrm{S}_{\mathrm{N}} 2^{\prime}$-type process can be achieved by using a vinylboronate complex with an allylic leaving group, thereby introducing a three-carbon motif and forming an allylic organoboron compound. This process works well for boranes, ${ }^{49}$ and the homologation was applied to achiral tertiary boronic esters by Smith et al..$^{50}$ When our research group applied this technique, homologation of enantioenriched tertiary boronic ester $\mathbf{5 1}$ with (3-chloroprop-1-en-1-yl)-lithium 52 afforded, after oxidation, allylic alcohol 53 in 68\% yield and $100 \%$ es, albeit in 1:1 dr (Scheme $10 \mathrm{a}){ }^{26}$ To obtain higher diastereoselectivity, we have found an $\mathrm{S}_{\mathrm{N}} 2$-type displacement of chloride to be preferential since the configurationally labile organolithium is then able to undergo a dynamic kinetic resolution. In this case, the homologation of 15 with 1-chloroallyllithium 54 afforded the allylic boronic ester 55 in 73\% yield, $100 \%$ es and $24: 1 \mathrm{dr}$ (Scheme 10b). ${ }^{42}$

We envisaged that if we could identify a method for promoting a 1,3-borotropic rearrangement of the products, we would have access 
a)

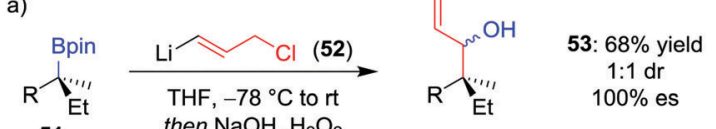

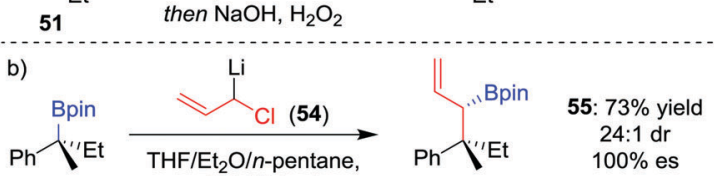
$15-120^{\circ} \mathrm{C}$ to $r$

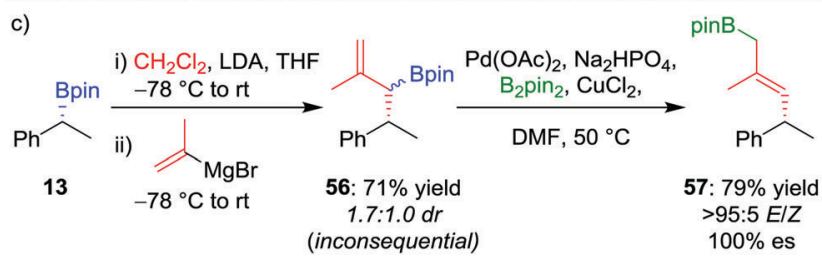

Scheme 10 Three-carbon homologations of enantioenriched boronic esters, and the palladium-catalyzed 1,3-borotropic shift. $\mathrm{R}=\mathrm{CH}_{2} \mathrm{CH}_{2} \mathrm{Ph}$.

to terminal allylic boronic esters for further functionalization. ${ }^{48}$ However, this posed two challenges: firstly, the homologation of a chiral secondary boronic ester by using 1-chloro-allyllithium leads to a mixture of under- and over-homologated products; secondly, allylic pinacol boronic ester products are stable to 1,3-borotropic rearrangements, unlike their propylene glycol analogues. ${ }^{50 b, 51}$

Consequently, to address the first challenge, we adopted a two-step, one-pot protocol involving a one-carbon Matteson homologation followed by in situ treatment with vinyl magnesium bromide (Scheme 10c). ${ }^{48}$ Next, to facilitate the 1,3-rearrangement, we discovered that palladium catalysis in the presence of base and $\mathrm{B}_{2} \mathrm{pin}_{2}$ gave access to a Bpin-bound $\pi$-allyl-Pd ${ }^{\mathrm{II}}$ complex 60 (Fig. 5) which, upon reductive elimination, affords the desired product with excellent $E$ selectivity. The three-carbon homologation of secondary boronic ester 13 was found to afford 57 in 61\% yield (over 2 steps), $E / Z>95: 5$ and $100 \%$ es (Scheme 10c). To demonstrate the synthetic utility of the three-carbon homologation, we applied our methodology to the synthesis of a fragment of $(+)$-jasplakinolide.

\subsection{Lithiation-borylation homologations}

Owing to the stereospecific nature of the 1,2-metallate rearrangement, there is the opportunity to create two or more

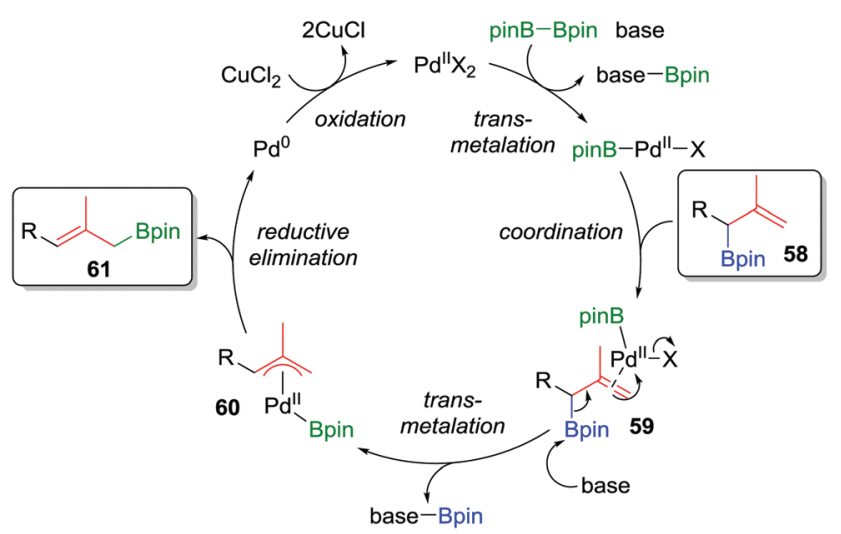

Fig. 5 Proposed mechanism of the palladium-catalyzed 1,3-rearrangement of allylic boronic esters used in a two-step three-carbon homologation. configurationally well-defined contiguous stereocentres with high fidelity through a stereoselective homologation. We have realised this through the application of our lithiationborylation methodology, ${ }^{6,12,13 d, 52}$ in which a configurationally stable carbenoid, possessing a suitable leaving group, is added to a boronic ester to ultimately form a new carbon-carbon bond at a stereogenic centre. Lithiated carbamate $\mathbf{6 3}$ can be formed by the deprotonation of the parent carbamate using secbutyllithium in the presence of (-)-sparteine (Scheme 11a). ${ }^{53}$ The addition of enantioenriched boronic ester 62 leads to the formation of boronate complex 64 which, following 1,2-metallate rearrangement and oxidation of the boronic ester, affords alcohol 66 in $82 \%$ yield, with $>98: 2$ er and 96:4 dr. ${ }^{52 a}$ Simply changing the enantiomer of sparteine (or using O'Brien's (+)-sparteine surrogate ${ }^{54}$ ) during the lithiation gives access to the other diastereomer of the product (70). We have subsequently applied this methodology to install two contiguous stereocentres in the synthesis of a range of natural products. ${ }^{37 a, 55}$
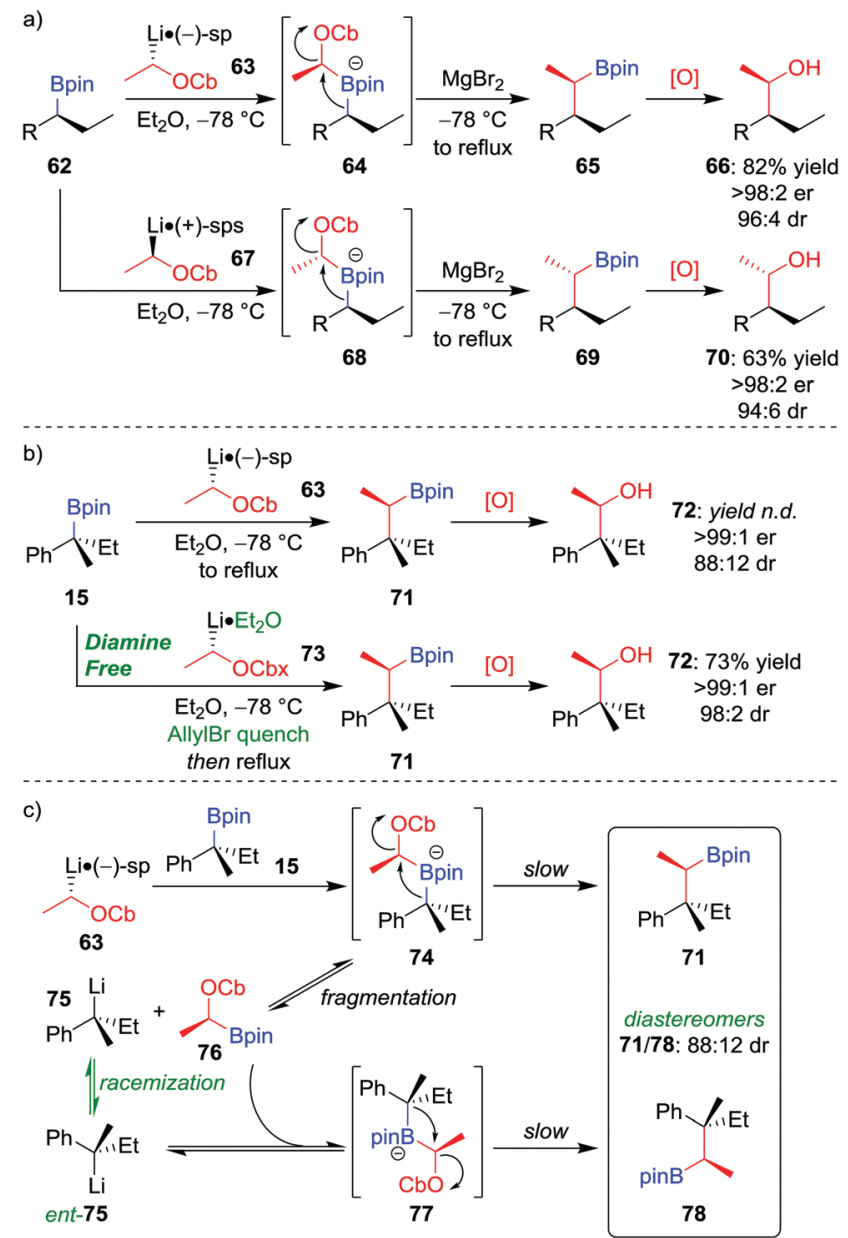

Scheme 11 Lithiation-borylation reactions of enantioenriched boronic esters to form (a) tertiary-tertiary and (b) quaternary-tertiary contiguous stereogenic centres. (c) Proposed mechanism for the generation of diastereomers in the formation of quaternary-tertiary contiguous stereogenic centres by lithiationborylation. $\mathrm{OCb}=\mathrm{N}, \mathrm{N}$-diisopropylcarbamate, $\mathrm{OCbx}=3,3$-dimethyl-1-oxa-4azaspiro[4.5]decane-4-carboxylate, $\mathrm{sp}=$ sparteine, $\mathrm{sps}=$ sparteine surrogate, $\mathrm{R}=\mathrm{CH}_{2} \mathrm{CH}_{2} \mathrm{Ph}$, n.d. $=$ not determined. 
A modified method can be used to incorporate quaternary stereocentres. Application of the standard method, described above, to the homologation of tertiary boronic ester 15 (99:1 er) with lithiated carbamate 63 (98:2 er), gives alcohol 72 with a surprisingly low $\mathrm{dr}\left(88: 12\right.$; Scheme 11b). ${ }^{56}$ It was discovered that epimerization had occurred at the quaternary stereogenic centre derived from the boronic ester. Consequently, the origin of minor diastereomer $\mathbf{7 8}$ was proposed to result from fragmentation of the sterically crowded boronate complex, forming a new organolithium (75) which can racemize and recombine with boronic ester 76 (Scheme 11c). To suppress this racemization pathway, the procedure was adapted as follows: firstly, adding allyl bromide after formation of boronate complex $\mathbf{7 4}$ traps any subsequently formed fragmentation anion 75; secondly, using diamine-free conditions (73 obtained by tin-lithium exchange from the corresponding stannane) minimises steric hindrance of the lithiated carbamate, thus enabling boronate complex formation. Application of these conditions affords alcohol 72 in 73\% yield, $>99: 1$ er and 98:2 dr (Scheme 11b).

Extending these conditions further to the synthesis of contiguous quaternary-quaternary stereogenic centres was found

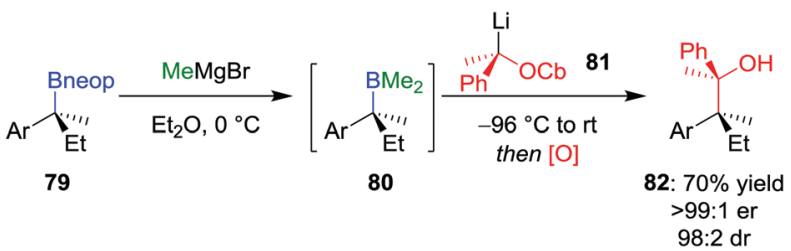

Scheme 12 Lithiation-borylation reactions of enantioenriched boronic esters to form quaternary-quaternary contiguous stereogenic centres. $\mathrm{OCb}=$ $\mathrm{N}, \mathrm{N}$-diisopropylcarbamate, $\mathrm{Ar}=\mathrm{p}-\mathrm{OMeC}_{6} \mathrm{H}_{4}$, neop = neopentyl glycol. to be even more challenging, with no 1,2-metallate rearrangement observed upon the addition of a tertiary boronic ester to a lithiated secondary carbamate. ${ }^{57}$ However, the reaction does proceed with mixed borane $\mathbf{8 0}$ (derived from in situ addition of $\mathrm{MeMgBr}$ to neopentyl glycol boronic ester 79) to afford, after oxidation, alcohol 82 in 70\% yield, $>99: 1$ er and 98:2 dr (Scheme 12). Notably, these mixed boranes complex with the benzylic lithium carbenoids with inversion of configuration at the carbenoid carbon atom. ${ }^{12}$ In addition to oxidation, amination of the borane intermediate using chloramine also worked well.

Using lithiation-borylation methodology, we have been able to develop a route to multiple contiguous stereocentres through an assembly-line process, without the requirement for multiple purifications. Nine iterative homologations, starting from boronic ester 83 and using lithiated benzoate ester $\mathbf{8 4}$ as the carbenoid (the OTIB group acts as a better leaving group when compared to $\mathrm{OCb}^{52 b}$ ), afforded boronic ester 85 in $58 \%$ yield with only two aqueous work-up steps and one purification (Scheme 13a). ${ }^{58}$ The all-anti isomer was found to adopt an irregular conformation, but by varying the enantiomer of $\mathbf{8 4}$ during the assembly-line process, the all-syn (86) and alternating syn-anti (87) isomers could be obtained, molecules that were found to adopt helical and linear conformations respectively. Assembly-line synthesis has also been applied using combinations of different types of homologation reactions. For example, incorporating sequential lithiation-borylation and Matteson homologation steps (see Section 4.1) followed by Morken's amination protocol (see Section 2.2) afforded 89 in $52 \%$ yield (Scheme 13b), an intermediate in the synthesis of (+)-kalkitoxin (90). ${ }^{59}$ Sequential homologations were also utilised in the
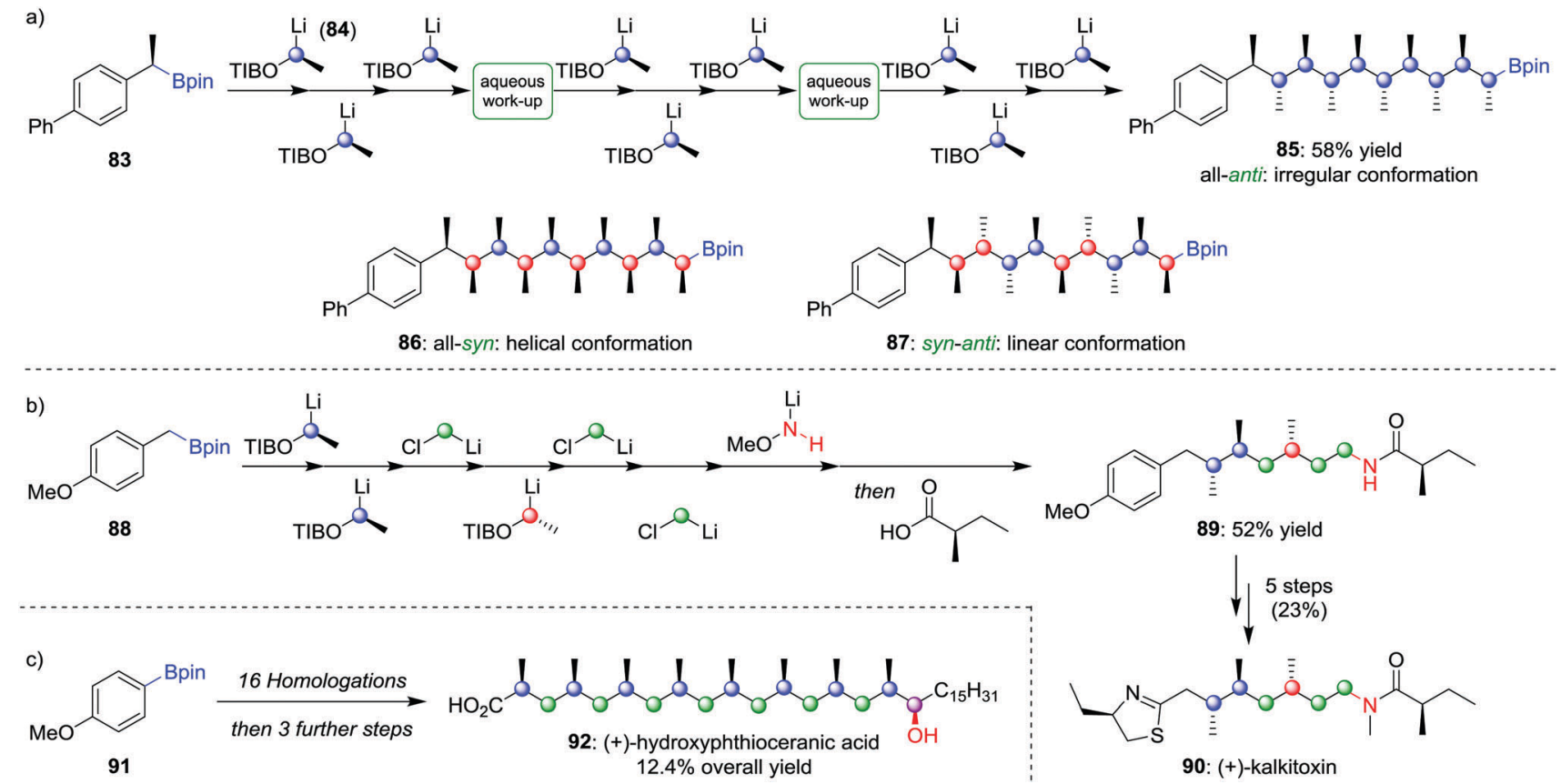

Scheme 13 Assembly-line synthesis used to form molecules with contiguous stereocentres by using multiple homologations. OTIB = 2,4,6triisopropylbenzoate. 
synthesis of (+)-hydroxyphthioceranic acid (92) from boronic ester 91 in $12.4 \%$ overall yield (Scheme 13c).

\subsection{Olefinations and alkynylations}

The selective formation of a single alkene isomer from a borane starting material was first reported by Zweifel and co-workers in $1967 .{ }^{60}$ In their seminal study, it was found that the addition of iodine to alkenyldialkylborane 93 (formed by hydroboration across an alkyne) results in the formation of iodonium ion $\mathbf{9 4}$, which, upon migration of an alkyl substituent of the boron, is opened to afford $\beta$-iodo-organoborane 95 (Scheme 14a). Deboronoiodination of this intermediate (usually facilitated by hydroxide or methoxide as a base) leads to cis-alkene $\mathbf{9 6 .}$ The reaction was subsequently expanded to the coupling of a boronic ester (98) with a vinyllithium (97) by Evans and co-workers (Scheme 14b). ${ }^{61}$ Owing to the stereospecific nature of the 1,2-metallate rearrangement, the reaction can be used to couple enantioenriched boronic esters with olefinic functional groups without loss of stereochemical integrity.

Our research group has shown that upon subjection of tertiary benzylic boronic esters to Zweifel olefination conditions, vinylmagnesium bromide adds not once but three times to the boronic ester, to form boronate complex 101 (Scheme 15a). ${ }^{42}$ The addition of four equivalents of vinylmagnesium bromide leads to complete conversion to this boronate complex, which, upon addition of iodine and $\mathrm{NaOMe} / \mathrm{MeOH}$, affords the olefination product 102 in $79 \%$ yield and $100 \%$ es.

In further studies within our group, we have identified that Zweifel olefination conditions can be applied to the homologation of a boronic ester to the corresponding methyl ketone using ethoxy vinyllithium (Scheme 15b). ${ }^{26,42}$ We have also applied this versatile reaction in synthesis - propenylation of 104 affords compound $\mathbf{1 0 5}$ as an intermediate in the synthesis of (-)-aplysin 106 (Scheme 15c), ${ }^{62}$ and an intramolecular Zweifel reaction of compound $\mathbf{1 0 7}$ was used in the synthesis of (-)-filiformin 109 (Scheme 15d). ${ }^{56}$
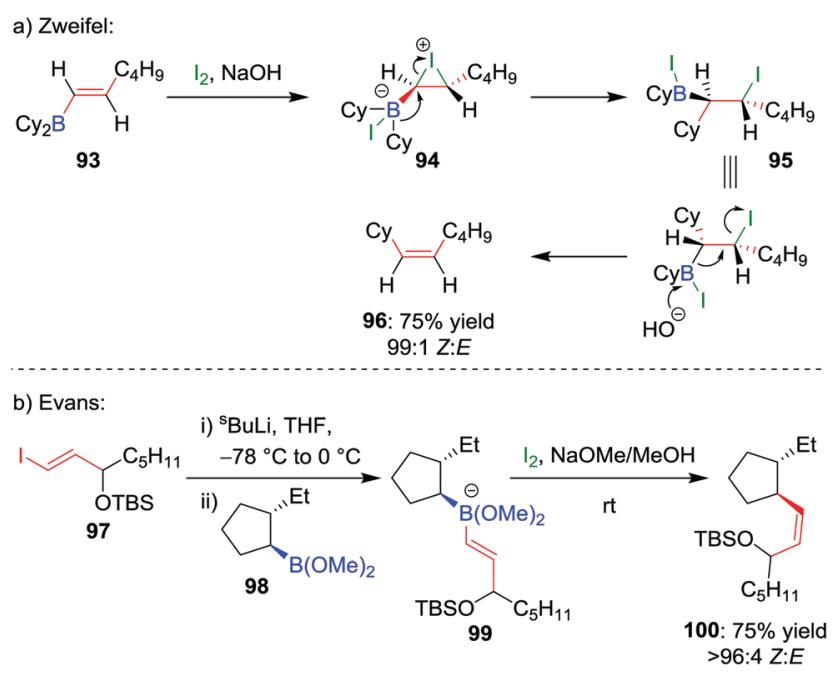

Scheme 14 Early examples of the Zweifel olefination reaction applied to boranes and boronic esters.
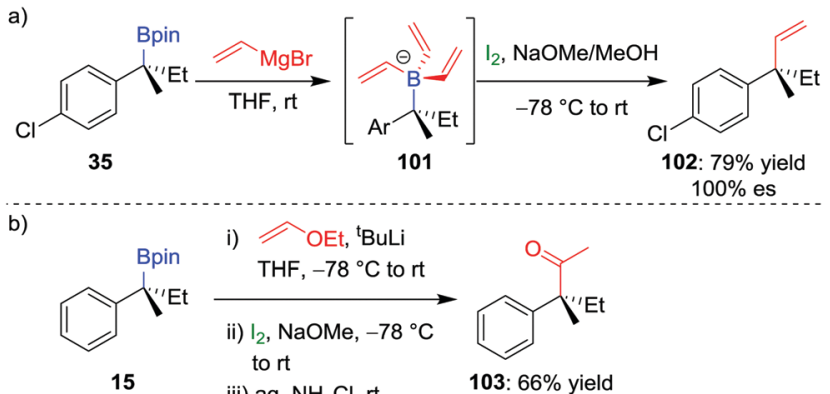

$$
\begin{aligned}
& \text { i) } \mathrm{OEt},{ }^{\mathrm{B} u L i} \\
& \mathrm{THF},-78^{\circ} \mathrm{C} \text { to rt } \\
& \text { ii) } \mathrm{I}_{2}, \mathrm{NaOMe},-78^{\circ} \mathrm{C} \\
& \text { to rt }
\end{aligned}
$$

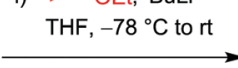

iii) aq. $\mathrm{NH}_{4} \mathrm{Cl}$, rt
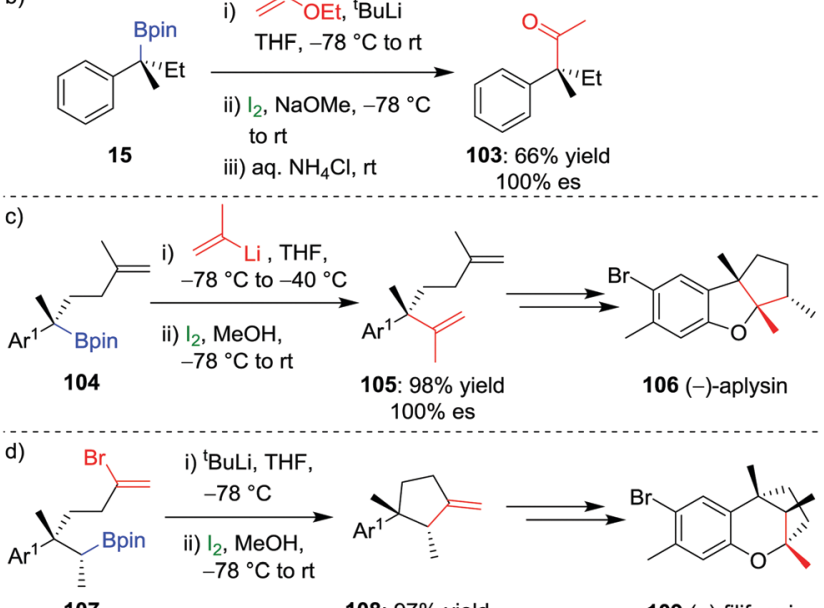

107

i) 'BuLi, THF, $100 \%$ es $100 \%$ es

Scheme 15 Application of the Zweifel olefination reaction to the functionalization of secondary and tertiary boronic esters. $\mathrm{Ar}=p-\mathrm{ClC}_{6} \mathrm{H}_{4}, \mathrm{Ar}^{1}=$ 2- $\mathrm{MeO}-4-\mathrm{Me}_{6}-\mathrm{C}_{6} \mathrm{H}_{3}$

The deboronoiodination of intermediate 95 occurs through an anti elimination process and gives access to the cis alkene. We reasoned that access to the alternative trans isomer would be facilitated through a syn elimination step. ${ }^{63}$ Replacing iodine with $\mathrm{PhSeCl}$ as the electrophile, followed by chemoselective oxidation of the $\beta$-selenoboronate intermediate 111 to the corresponding selenoxide 112, initiates syn elimination of selenium and boron (Scheme 16a). This protocol allowed the coupling of vinyllithium 114 (derived by lithium-halogen exchange from the parent vinylbromide) with boronic ester $\mathbf{2 8}$ to afford trans alkene 115 in 74\% yield, >98:2 E/Z selectivity and $100 \%$ es (Scheme 16b). ${ }^{64}$ Taken in combination with the
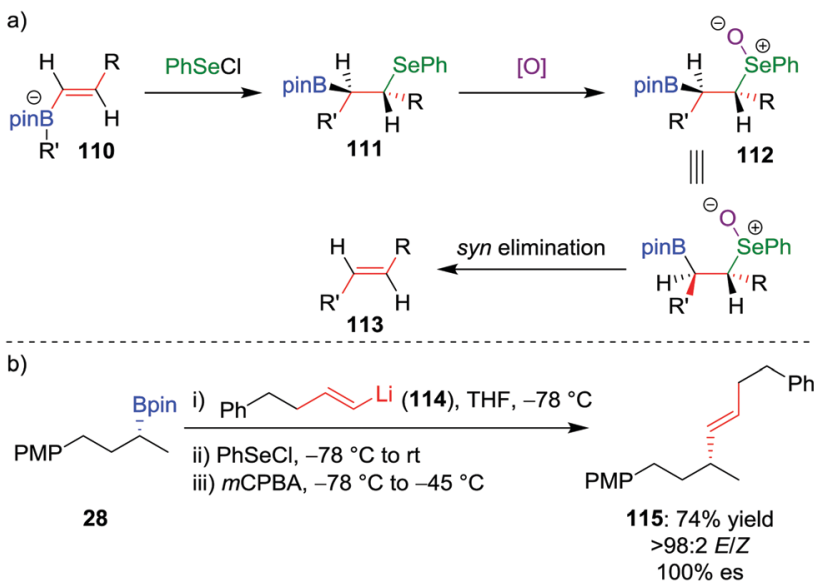

Scheme 16 Trans selective variant of the Zweifel olefination reaction. $\mathrm{PMP}=$ para-methoxyphenyl. 
Zweifel cis olefination, the two procedures provide stereodivergent access to either alkene isomer. ${ }^{65}$

Extension of olefination techniques to alkynylation is more challenging. The reaction of a metallated alkyne with a boronic ester is reversible, ${ }^{66}$ and, consequently, addition of iodine affords an iodoalkyne rather than the desired $\pi$-electrophilic activation of the alkynyl boronate. However, our group has shown that suitably functionalized alkenes, which can be coupled to boronic esters by using the Zweifel olefination, can give an alkyne through a subsequent 1,2-elimination. ${ }^{67}$ Deprotonation of vinylbromide by LDA and addition to enantioenriched secondary alkylboronic ester 116, followed by the addition of iodine in methanol, was found to afford 1,1-bromoalkyl-alkene 117 (Scheme 17a). A solution of crude vinyl bromide 117 was then treated with TBAF to achieve elimination and afford the desired alkyne 118 in 81\% overall yield and complete enantiospecificity.

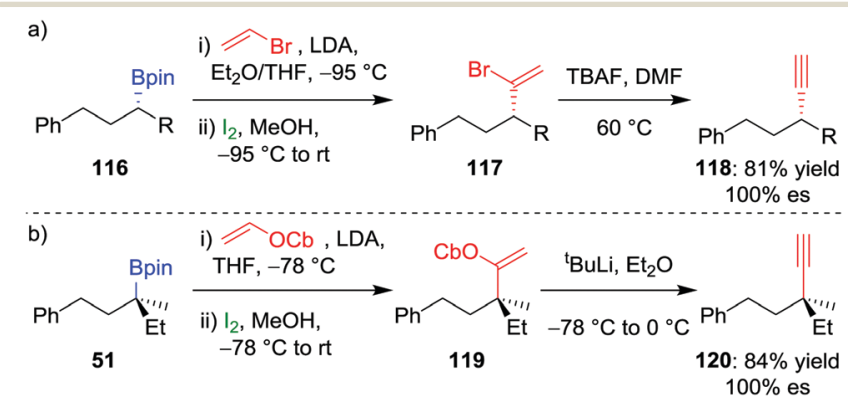

Scheme 17 Stereospecific alkynylation of secondary and tertiary boronic esters. $\mathrm{R}=\mathrm{CH}_{2} \mathrm{CH}\left(\mathrm{CH}_{3}\right)_{2}, \mathrm{OCb}=\mathrm{N}, \mathrm{N}$-diisopropylcarbamate.

For the alkynylation of tertiary boronic esters, we found that vinyl carbamate is a superior reagent, with the intermediate carbamoylalkylalkene 119 easily undergoing elimination by using tert-butyllithium to afford alkyne 120 in $84 \%$ yield and $100 \%$ es (Scheme 17b). Secondary benzylic boronic esters also undergo alkynylation by using vinyl carbamate, but the stoichiometry of the tert-butyllithium must be carefully controlled to prevent deprotonation at the benzylic carbon centre and a concomitant reduction in enantiospecificity.

\subsection{Addition to aldehydes and iminium ions}

During our studies into the reactivity of boronic esters, we observed that benzylic boronic esters react with aldehydes in the presence of CsF, albeit with only $43 \%$ es. ${ }^{68}$ Switching to a benzylic trifluoroborate salt $\mathbf{1 2 1}$ (obtained from the parent boronic ester in excellent yields and stereospecificity $)^{23}$ and using a catalytic amount of $\left[\{\operatorname{RhCl}(\operatorname{cod})\}_{2}\right]$ enabled the addition to aldehyde 122 to be conducted in $86 \%$ yield and $100 \%$ es, through a stereoretentive pathway (Scheme 18a). The retention of configuration was explained by invoking rhodium acting as a Lewis acid to simultaneously coordinate both the aldehyde and boron compound and facilitate carbon-carbon bond formation (Fig. 6). Both secondary and tertiary benzylic trifluoroborate salts participate in this reaction, and TEMPO-mediated oxidation of the products led to the corresponding ketone $\mathbf{1 2 4}$ without stereoerosion. Yun and co-workers extended this methodology
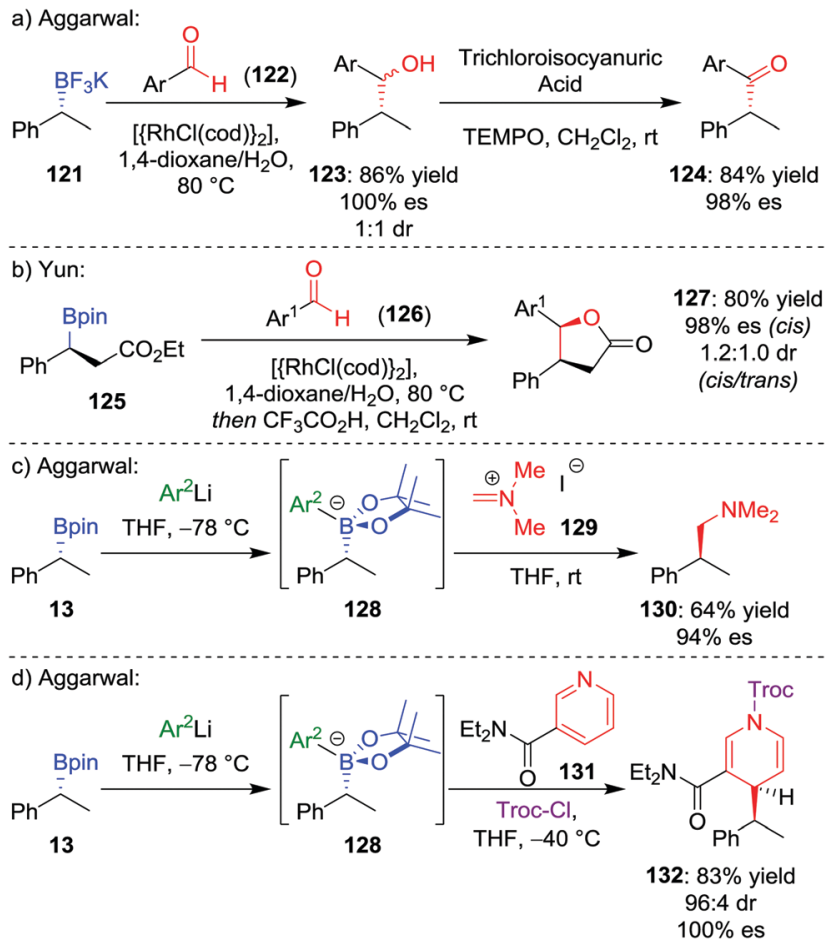

Scheme 18 Additions of trifluoroborate salts and boronate complexes to aldehydes and iminium ions. $\mathrm{Ar}=p-\mathrm{CF}_{3} \mathrm{C}_{6} \mathrm{H}_{4}, \mathrm{Ar}^{1}=p-\mathrm{NO}_{2} \mathrm{C}_{6} \mathrm{H}_{4}, \mathrm{Ar}^{2}=$ 3,5- $\left(\mathrm{CF}_{3}\right)_{2} \mathrm{C}_{6} \mathrm{H}_{3}$, Troc- $\mathrm{Cl}=$ 2,2,2-trichloroethyl chloroformate.

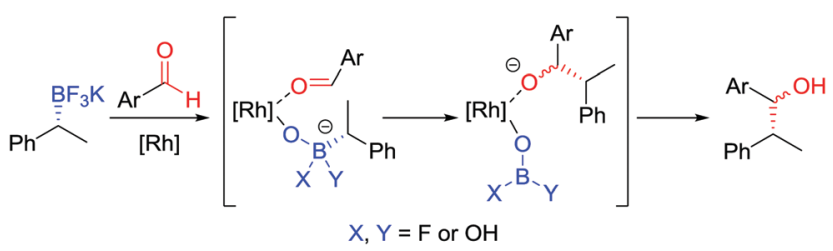

Fig. 6 Proposed mechanism for the stereospecific addition of trifluoroborate salts to aldehydes, catalysed by $\left[\{\mathrm{RhCl}(\mathrm{cod})\}_{2}\right]$.

to form $\gamma$-butyrolactones 127 , using $\mathrm{KHF}_{2}$ as an additive to enable the reaction of benzylic boronic esters, albeit with moderate diastereocontrol (Scheme 18b). ${ }^{69}$

In addition to the reaction of trifluoroborate salts, our group has also shown that boronate complexes (see Section 2.3) react with iminium ions to form $\mathrm{C}-\mathrm{C}$ bonds without loss of stereochemical integrity. Reaction of boronate 128 with commercially available Eschenmoser's salt 129 gives tertiary amine 130 in $64 \%$ yield and $94 \%$ es (Scheme 18c). ${ }^{28}$ Benzylic boronate complexes also react with cyclic iminium ions - the addition of boronate 128 to pyridine $\mathbf{1 3 1}$ (activated using Troc-Cl) affords dihydropyridine 132 in 83\% yield, 96:4 dr and 100\% es (Scheme 18d). ${ }^{70}$ Activated quinolines and dihydroisoquinolines can also be used as electrophiles.

\section{$4.6 \mathrm{sp}^{2}-\mathrm{sp}^{3}$ coupling reactions}

The formation of key $\mathrm{C}-\mathrm{C}$ bonds by cross-coupling reactions, such as the Suzuki-Miyaura reaction, is one of the most established methods in modern synthesis. ${ }^{4,71}$ However, the 
Suzuki-Miyaura reaction is largely limited to the formation of $\mathrm{sp}^{2}-\mathrm{sp}^{2}$ bonds, owing to problems of slow transmetalation and competing $\beta$-hydride elimination when extended to coupling of $\mathrm{sp}^{3}$ carbon centres. ${ }^{72}$ Specifically, $\beta$-hydride elimination and subsequent hydropalladation leads to racemization of stereogenic centres and loss of stereochemical information. Accordingly, significant research has focussed on the coupling of $\mathrm{sp}^{3}$ organometallic nucleophiles within recent years. ${ }^{73}$

The first general stereospecific coupling of an enantioenriched secondary benzylic boronic ester was achieved by Crudden and co-workers in $2009 ;^{74}$ using $\left[\mathrm{Pd}_{2}\left(\mathrm{dba}_{3}\right] / \mathrm{PPh}_{3}\right.$ as the catalyst, boronic ester ent-13 was coupled in a stereoretentive manner with aryl iodide 133 in $62 \%$ yield and $91 \%$ es (Scheme 19a). Crucially, the use of $\mathrm{Ag}_{2} \mathrm{O}$ as the base, which increases the rate of the transmetalation step, was found to be critical to obtaining high yields and high levels of enantiospecificity. ${ }^{75}$ Crudden and co-workers have further extended their methodology to enable the coupling of dibenzylic boronic esters; in this case, neopentyl glycol boronic ester $\mathbf{1 3 5}$ was coupled to give triarylmethane $\mathbf{1 3 6}$ in high yield and enantiospecificity (Scheme 19b). ${ }^{76}$ Liao and co-workers found that although dibenzylic boronic esters coupled with retention of configuration, the corresponding trifluoroborates coupled with inversion of configuration (Scheme 19c). ${ }^{77}$

The first report of an enantiospecific Suzuki-Miyaura coupling of an unactivated dialkylboron compound was reported by Biscoe and co-workers in 2014. ${ }^{78}$ Stereoinvertive coupling of enantioenriched trifluoroborate salt 140 with chlorobenzene 141 was achieved using preformed $\mathrm{P}^{t} \mathrm{Bu}_{3}$-ligated palladium precatalyst $142,{ }^{79}$ affording 143 in $64 \%$ yield and $94 \%$ es (Scheme 19d). The bulky, electron-rich

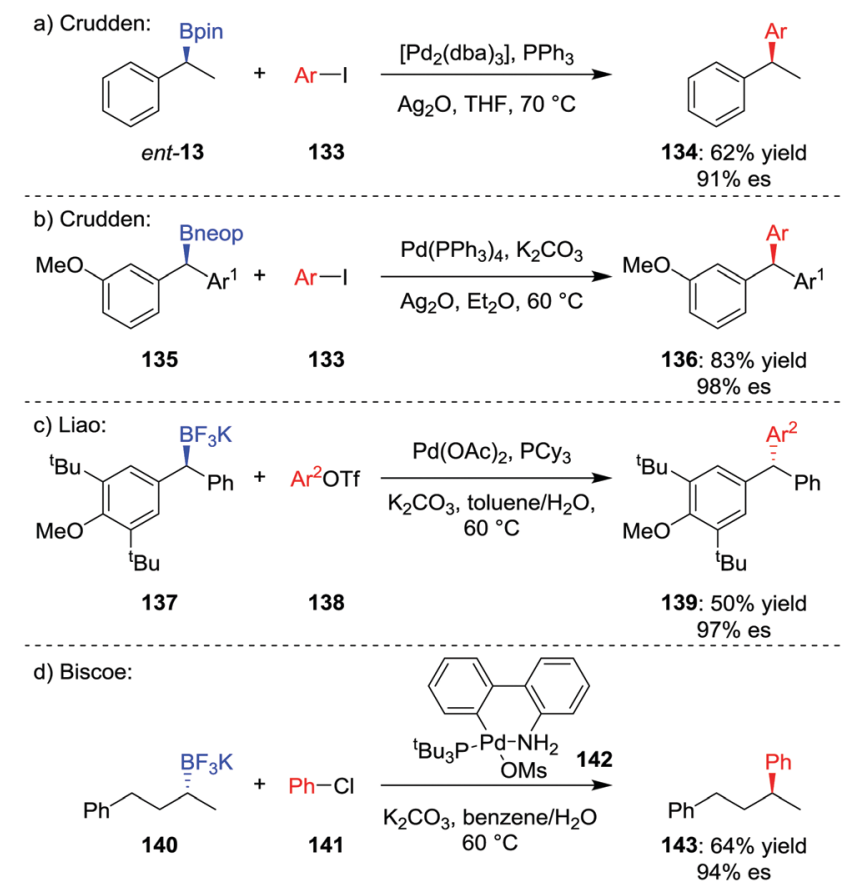

Scheme 19 Enantiospecific transition-metal-mediated $\mathrm{sp}^{2}-\mathrm{sp}^{3}$ crosscoupling of unactivated secondary boronic esters and trifluoroborate salts $\mathrm{Ar}=p-\mathrm{ClC}_{6} \mathrm{H}_{4}, \mathrm{Ar}^{1}=p-\mathrm{CH}_{3} \mathrm{CH}_{2} \mathrm{C}_{6} \mathrm{H}_{4}, \mathrm{Ar}^{2}=p-\mathrm{CH}_{3} \mathrm{C}_{6} \mathrm{H}_{4}, \mathrm{Ms}=\mathrm{CH}_{3} \mathrm{SO}_{2}$, neop $=$ neopentyl glycol.

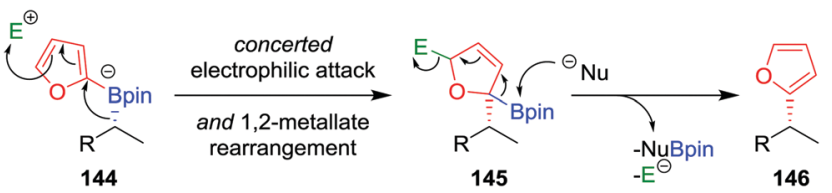

Fig. 7 Mechanism for the stereospecific transition-metal-free coupling of boronic esters with furan. $\mathrm{E}^{+}=$electrophile, $\mathrm{Nu}^{-}=$nucleophile.

phosphine ligand is used to suppress $\beta$-hydride elimination and subsequent isomerization, as reported by the groups of Dreher and Molander. ${ }^{80}$

As a consequence of the difficulties associated with transitionmetal-mediated $\mathrm{sp}^{2}-\mathrm{sp}^{3}$ coupling reactions, particularly the inability to couple tertiary boronic esters under current protocols, we reasoned that the desired $\mathrm{C}-\mathrm{C}$ bond could be formed using an alternative transition-metal-free route. In our approach, we proposed that activation of the aromatic ring of boronate complex 144 by an electrophile (in an analogous fashion to the activation of the alkene in a Zweifel reaction; see Section 4.4) would facilitate the desired 1,2-metallate rearrangement (Fig. 7). ${ }^{81}$ Upon nucleophilic attack at the boron centre, the compound would re-aromatize with the elimination of the electrophile, affording the desired coupled product $\mathbf{1 4 6}$ in a stereoretentive fashion.

We initially tested this reaction manifold on the coupling of 2-lithiofuran (formed by deprotonation of furan by $n$-butyllithium) with enantioenriched boronic ester ent-20 (Scheme 20a). ${ }^{81 a, b}$ Following formation of boronate complex 147, addition of $\mathrm{N}$-bromosuccinimide as the electrophile facilitates the desired coupling in $91 \%$ yield and $100 \%$ es. Significantly, the procedure

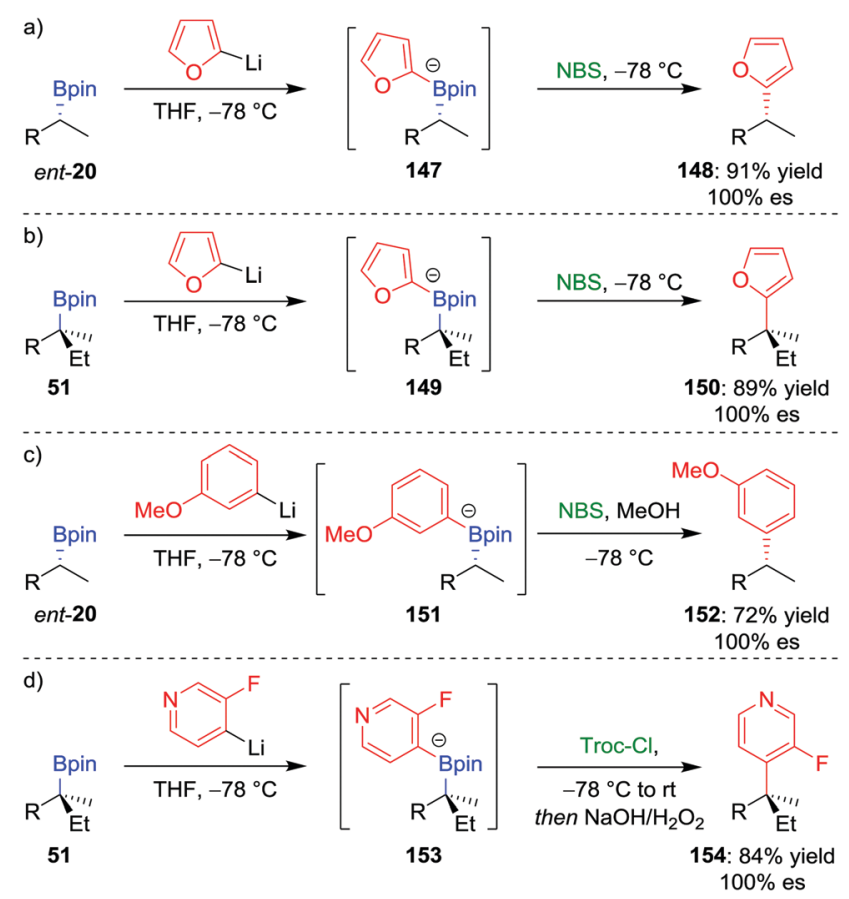

Scheme 20 Transition-metal-free cross-coupling reactions of secondary and tertiary boronic esters. $\mathrm{R}=\mathrm{CH}_{2} \mathrm{CH}_{2} \mathrm{Ph}, \mathrm{NBS}=\mathrm{N}$-bromosuccinimide, Troc- $\mathrm{Cl}=$ 2,2,2-trichloroethyl chloroformate. 
can be applied to tertiary boronic esters (Scheme 20b), which are not good substrates for the alternative transition-metalmediated coupling reactions discussed above. The reaction was also found to be applicable to coupling six-membered aromatics: the reaction of lithiated meta-methoxybenzene with boronic ester ent-20 gave the coupled product in $72 \%$ yield and $100 \%$ es (Scheme 20c). It was found that an electron-donating group at the meta position of the aromatic ring was essential to prevent reaction of the boronate complex with the electrophile at the $\mathrm{sp}^{3}$ carbon-boron bond ( $c f$. Section 2.3).

The scope of this coupling reaction has been extended to include N-heteroaromatic compounds. ${ }^{82}$ Reminiscent of the reactions involving the addition of nucleophilic boronate complexes to activated cyclic iminium ions (see Section 4.5), ${ }^{70}$ we proposed that activation of the nitrogen atom of boronate complex 153, through acylation with Troc-Cl, would facilitate 1,2-metallate rearrangement (Scheme 20d). Oxidation of the resulting boronic ester facilitated rearomatization to afford coupled product 154 in $84 \%$ yield and with complete retention of configuration.

In a further extension to this methodology, we reasoned that using a carbon-based electrophile would afford intermediate 145 (Fig. 7), but this intermediate would not be susceptible to elimination. Oxidation of the boronic ester and subsequent rearomatization would instead yield a three-component coupling product. To test this strategy, ${ }^{83}$ we applied electrophilic trifluoromethylation to obtain 2,5-disubstituted furans containing a key $\mathrm{CF}_{3}$ group. For example, trifluoromethylation of boronate complex 155, through the addition of Umemoto's reagent, afforded intermediate 156, which could be oxidised using iodine to give the three-component coupled product 157 in $72 \%$ yield and $100 \%$ es (Scheme 21a). Tertiary boronic esters could also be used, but the oxidation step was found to be more challenging: only one diastereomer of $\mathbf{1 6 0}$ could be oxidised to the threecomponent coupled product 159 under the reaction conditions (Scheme 21b). Mechanistic studies determined that the reaction proceeds through a radical propagation cycle; the trifluoromethyl radical was observed by EPR spectroscopy using a spin trapping experiment.

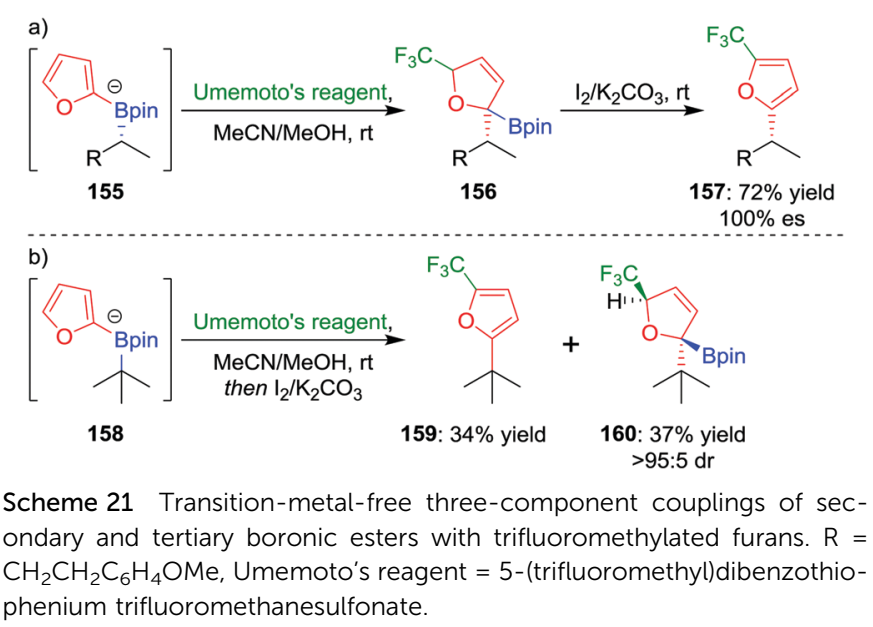

\section{Conclusions and outlook}

The capability of boronic esters to undergo stereospecific transformations facilitates access to a wide range of enantioenriched building blocks for synthesis. Consequently, new asymmetric methods to create chiral boronic esters represent an efficient route towards diverse functionality. These two research areas are currently developing synergistically to form a major pillar in modern asymmetric synthesis. ${ }^{26,42,84}$

For the main part, the stereospecificity of these reactions results from a 1,2-metallate rearrangement of a boronate complex, in which the carbon-boron $\sigma$-bond migrates with retention of configuration. This 1,2-metallate rearrangement has been manipulated to enable a range of new bond-forming reactions, most notably, oxidation, amination, homologation, olefination, alkynylation and arylation reactions. However, in some cases new reactivity pathways for boronic esters have been uncovered: the reaction of nucleophilic boronate complexes with electrophiles, a transformation that occurs with inversion of configuration; complexation to water molecules in protodeboronation; boron-palladium transmetalation without significant $\beta$-hydride elimination.

Broad though these methods are, access to certain functional groups remains elusive. Considering homologation reactions, if the desired nucleophile for attacking the boronic ester is too stabilised, it simply decomplexes from the boron centre rather than undergo 1,2-migration. This reluctance to form stable boronate complexes has thwarted some attempts at developing novel functionalization reactions of boronic esters, such as direct alkynylation by using alkynyl anions (see Section 4.4). We anticipate that new strategies to circumvent these problems will provide access to new functionalities, notable current remaining challenges being stereospecific transformations to thiols, nitriles, carboxylic acids, nitro compounds, trifluoromethyl groups, and phosphorus functional groups.

Finally, the stereospecific coupling of secondary and tertiary boronic esters to aromatic groups is a field that has received considerable interest over the last decade, owing to the synthetic utility of the products. Some progress has been made, but further developments to expand its generality are still required. One significant transformation that remains to be achieved is a general traceless $\mathrm{sp}^{3}-\mathrm{sp}^{3}$ coupling reaction between an enantioenriched boronic ester and a suitable coupling partner (such as an alkyl halide). The development of such a method would further extend the broad array of transformations available to enantioenriched boronic esters and would be a major step forward for the field of asymmetric synthesis.

\section{Acknowledgements}

We acknowledge financial support from EPSRC (EP/I038071/1) the European Research Council (FP7, ERC grant no. 670668) and the University of Bristol. We thank Dr Eddie L. Myers for valuable discussions.

\section{References}

1 (a) H. C. Brown and G. Zweifel, J. Am. Chem. Soc., 1961, 83, 486-487; see also: (b) G. Zweifel and H. C. Brown, J. Am. Chem. Soc., 1964, 86, 
393-397; (c) H. C. Brown, M. C. Desai and P. K. Jadhav, J. Org. Chem., 1982, 47, 5065-5069.

2 H. C. Brown and B. Singaram, Acc. Chem. Res., 1988, 21, 287-293.

3 (a) A. J. J. Lennox and G. C. Lloyd-Jones, Chem. Soc. Rev., 2014, 43, 412-443; (b) D. S. Matteson, J. Organomet. Chem., 1999, 581, 51-65.

4 (a) S. Kotha, K. Lahiri and D. Kashinath, Tetrahedron, 2002, 58, 9633-9695; (b) J. Magano and J. R. Dunetz, Chem. Rev., 2011, 111, 2177-2250.

5 D. S. Matteson, Chem. Rev., 1989, 89, 1535-1551.

6 (a) D. Leonori and V. K. Aggarwal, Acc. Chem. Res., 2014, 47, 3174-3183; (b) D. Leonori and V. K. Aggarwal, in Synthesis and Application of Organoboron Compounds, ed. E. Fernández and A. Whiting, Springer International Publishing, Cham, 2015, pp. 271-295.

7 (a) C. M. Crudden and D. Edwards, Eur. J. Org. Chem., 2003, 4695-4712; (b) A.-M. Carroll, T. P. O'Sullivan and P. J. Guiry, Adv. Synth. Catal., 2005, 347, 609-631.

8 (a) S. Lee and J. Yun, in Synthesis and Application of Organoboron Compounds, ed. E. Fernández and A. Whiting, Springer International Publishing, Cham, 2015, pp. 73-92; (b) J. A. Schiffner, K. Müther and M. Oestreich, Angew. Chem., Int. Ed., 2010, 49, 1194-1196.

9 (a) J. R. Coombs and J. P. Morken, Angew. Chem., Int. Ed., 2016, 55, 2636-2649; (b) J. Takaya and N. Iwasawa, ACS Catal., 2012, 2, 1993-2006.

10 (a) H. K. Scott and V. K. Aggarwal, Chem. - Eur. J., 2011, 17, 13124-13132; (b) T. Chinnusamy, K. Feeney, C. G. Watson, D. Leonori and V. K. Aggarwal, in Comprehensive Organic Synthesis, ed. G. A. Molander and P. Knochel, Elsevier, Oxford, 2nd edn, 2014, vol. 7, pp. 692-718.

11 H. C. Brown and G. Zweifel, J. Am. Chem. Soc., 1961, 83, 2544-2551.

12 J. L. Stymiest, V. Bagutski, R. M. French and V. K. Aggarwal, Nature, 2008, 456, 778-782.

13 (a) A. G. Davies and B. P. Roberts, J. Chem. Soc. B, 1967, 17-22; (b) G. W. Burton and K. U. Ingold, J. Am. Chem. Soc., 1981, 103, 6472-6477; (c) L. Shu and Y. Shi, J. Org. Chem., 2000, 65, 8807-8810; (d) V. Bagutski, R. M. French and V. K. Aggarwal, Angew. Chem., Int. Ed., 2010, 49, 5142-5145.

14 G. W. Kabalka, T. M. Shoup and N. M. Goudgaon, Tetrahedron Lett., 1989, 30, 1483-1486.

15 (a) P. Fontani, B. Carboni, M. Vaultier and G. Maas, Synthesis, 1991, 605-609; see also: (b) M. M. Hussain, H. Li, N. Hussain, M. Ureña, P. J. Carroll and P. J. Walsh, J. Am. Chem. Soc., 2009, 131, 6516-6524.

16 (a) C. N. Farthing and S. P. Marsden, Tetrahedron Lett., 2000, 41, 4235-4238; (b) H.-S. Sim, X. Feng and J. Yun, Chem. - Eur. J., 2009, 15, 1939-1943.

17 S. Radomkit and A. H. Hoveyda, Angew. Chem., Int. Ed., 2014, 53, 3387-3391.

18 H. C. Brown, K.-W. Kim, M. Srebnik and S. Bakthan, Tetrahedron, 1987, 43, 4071-4078.

19 (a) H. C. Brown, A. Suzui, S. Sonao, M. Itoh and M. M. Midland, J. Am. Chem. Soc., 1971, 93, 4329-4330; (b) H. C. Brown, M. M. Midland and A. B. Levy, J. Am. Chem. Soc., 1972, 94, 2114-2115.

20 H. C. Brown, K. W. Kim, T. E. Cole and B. Singaram, J. Am. Chem. Soc., 1986, 108, 6761-6764.

21 S. N. Mlynarski, A. S. Karns and J. P. Morken, J. Am. Chem. Soc., 2012, 134, 16449-16451.

22 V. Bagutski, T. G. Elford and V. K. Aggarwal, Angew. Chem., Int. Ed., 2011, 50, 1080-1083.

23 V. Bagutski, A. Ros and V. K. Aggarwal, Tetrahedron, 2009, 65, 9956-9960.

24 D. S. Matteson and G. Y. Kim, Org. Lett., 2002, 4, 2153-2155.

25 B. J. Kim and D. S. Matteson, Angew. Chem., Int. Ed., 2004, 43, 3056-3058.

26 A. P. Pulis, D. J. Blair, E. Torres and V. K. Aggarwal, J. Am. Chem. Soc., 2013, 135, 16054-16057.

27 For other examples of chiral organometallic nucleophiles, see: (a) A. Boudier and P. Knochel, Tetrahedron Lett., 1999, 40, 687-690; (b) R. W. Hoffmann, B. Hölzer, O. Knopff and K. Harms, Angew. Chem., Int. Ed., 2000, 39, 3072-3074; (c) T. Thaler, B. Haag, A. Gavryushin, K. Schober, E. Hartmann, R. M. Gschwind, H. Zipse, P. Mayer and P. Knochel, Nat. Chem., 2010, 2, 125-130.

28 R. Larouche-Gauthier, T. G. Elford and V. K. Aggarwal, J. Am. Chem. Soc., 2011, 133, 16794-16797.

29 (a) H. C. Brown and C. F. Lane, J. Chem. Soc. D, 1971, 521-522; (b) H. C. Brown, N. R. De Lue, G. W. Kabalka and H. C. Hedgecock, J. Am. Chem. Soc., 1976, 98, 1290-1291; (c) H. C. Brown and C. F. Lane, Tetrahedron, 1988, 44, 2763-2772.
30 C. Sandford, R. Rasappan and V. K. Aggarwal, J. Am. Chem. Soc., 2015, 137, 10100-10103.

31 (a) H. Mayr and M. Patz, Angew. Chem., Int. Ed. Engl., 1994, 33, 938-957; (b) H. Mayr, B. Kempf and A. R. Ofial, Acc. Chem. Res., 2003, 36, 66-77; (c) G. Berionni, A. I. Leonov, P. Mayer, A. R. Ofial and H. Mayr, Angew. Chem., Int. Ed., 2015, 54, 2780-2783.

32 K. Feeney, G. Berionni, H. Mayr and V. K. Aggarwal, Org. Lett., 2015, 17, 2614-2617.

33 H. C. Brown and K. J. Murray, Tetrahedron, 1986, 42, 5497-5504.

34 S. Nave, R. P. Sonawane, T. G. Elford and V. K. Aggarwal, J. Am. Chem. Soc., 2010, 132, 17096-17098.

35 M. J. Hesse, C. P. Butts, C. L. Willis and V. K. Aggarwal, Angew. Chem., Int. Ed., 2012, 51, 12444-12448.

36 (a) R. Rasappan and V. K. Aggarwal, Nat. Chem., 2014, 6, 810-814; see also: (b) D. Pozzi, E. M. Scanlan and P. Renaud, J. Am. Chem. Soc., 2005, 127, 14204-14205; (c) G. Povie, G. Villa, L. Ford, D. Pozzi, C. H. Schiesser and P. Renaud, Chem. Commun., 2010, 46, 803-805.

37 (a) T. G. Elford, S. Nave, R. P. Sonawane and V. K. Aggarwal, J. Am. Chem. Soc., 2011, 133, 16798-16801; (b) S. Roesner, J. M. Casatejada, T. G. Elford, R. P. Sonawane and V. K. Aggarwal, Org. Lett., 2011, 13, 5740-5743; (c) V. K. Aggarwal, L. T. Ball, S. Carobene, R. L. Connelly, M. J. Hesse, B. M. Partridge, P. Roth, S. P. Thomas and M. P. Webster, Chem. Commun., 2012, 48, 9230-9232; (d) S. Roesner and V. K. Aggarwal, Can. J. Chem., 2012, 90, 965-974; (e) S. Roesner, D. J. Blair and V. K. Aggarwal, Chem. Sci., 2015, 6, 3718-3723.

38 S. P. Thomas, R. M. French, V. Jheengut and V. K. Aggarwal, Chem. Rec., 2009, 9, 24-39.

39 K. M. Sadhu and D. S. Matteson, Organometallics, 1985, 4, 1687-1689. 40 (a) H. C. Brown, S. M. Singh and M. V. Rangaishenvi, J. Org. Chem., 1986, 51, 3150-3155; (b) T. J. Michnick and D. S. Matteson, Synlett, 1991, 631-632; (c) R. Soundararajan, G. Li and H. C. Brown, Tetrahedron Lett., 1994, 35, 8957-8960.

41 (a) D. S. Matteson and D. Majumdar, Organometallics, 1983, 2, 1529-1535; (b) H. C. Brown, T. Imai, P. T. Perumal and B. Singaram, J. Org. Chem., 1985, 50, 4032-4036; (c) D. S. Matteson, Tetrahedron, 1998, 54, 10555-10607.

42 R. P. Sonawane, V. Jheengut, C. Rabalakos, R. Larouche-Gauthier, H. K. Scott and V. K. Aggarwal, Angew. Chem., Int. Ed., 2011, 50, 3760-3763.

43 (a) M. C. Elliott, K. Smith, D. H. Jones, A. Hussain and B. A. Saleh, J. Org. Chem., 2013, 78, 3057-3064; (b) M. C. Elliott and K. Smith, Organometallics, 2013, 32, 4878-4881; see also: (c) A. Bottoni, M. Lombardo, A. Neri and C. Trombini, J. Org. Chem., 2003, 68, 3397-3405.

44 A. Chen, L. Ren and C. M. Crudden, J. Org. Chem., 1999, 64, 9704-9710.

45 (a) M. W. Rathke, E. Chao and G. Wu, J. Organomet. Chem., 1976, 122, 145-149; (b) D. S. Matteson, H.-W. Man and O. C. Ho, J. Am. Chem. Soc., 1996, 118, 4560-4566.

46 K. R. Fandrick, J. A. Mulder, N. D. Patel, J. Gao, M. Konrad, E. Archer, F. G. Buono, A. Duran, R. Schmid, J. Daeubler, J.-N. Desrosiers, X. Zeng, S. Rodriguez, S. Ma, B. Qu, Z. Li, D. R. Fandrick, N. Grinberg, H. Lee, T. Bosanac, H. Takahashi, Z. Chen, A. Bartolozzi, P. Nemoto, C. A. Busacca, J. J. Song, N. K. Yee, P. E. Mahaney and C. H. Senanayake, J. Org. Chem., 2015, 80, 1651-1660.

47 D. S. Matteson and E. C. Beedle, Tetrahedron Lett., 1987, 28, 4499-4502.

48 P. J. Unsworth, D. Leonori and V. K. Aggarwal, Angew. Chem., Int. Ed., 2014, 53, 9846-9850.

49 (a) G. Zweifel, A. Horng and J. T. Snow, J. Am. Chem. Soc., 1970, 92, 1427-1429; (b) G. Zweifel and A. Horng, Synthesis, 1973, 672-674.

50 (a) K. Smith, M. C. Elliott and D. H. Jones, J. Org. Chem., 2013, 78, 9526-9531 for examples starting from a vinylboronic ester, see: (b) M. Lombardo, S. Morganti, M. Tozzi and C. Trombini, Eur. J. Org. Chem., 2002, 2823-2830; (c) F. Possémé, M. Deligny, F. Carreaux and B. Carboni, J. Org. Chem., 2007, 72, 984-989 for an example of a direct enantioselective $\mathrm{S}_{\mathrm{N}} 2^{\prime}$-allylic alkylation without formation of the boronate complex, see: (d) L. Carosi and D. G. Hall, Angew. Chem., Int. Ed., 2007, 46, 5913-5915.

51 H. C. Brown, M. V. Rangaishenvi and S. Jayaraman, Organometallics, 1992, 11, 1948-1954.

52 (a) J. L. Stymiest, G. Dutheuil, A. Mahmood and V. K. Aggarwal, Angew. Chem., Int. Ed., 2007, 46, 7491-7494; (b) R. Larouche-Gauthier, C. J. Fletcher, I. Couto and V. K. Aggarwal, Chem. Commun., 2011, 47, 
12592-12594 for an alternative approach to the generation of contiguous stereocentres using $\alpha$-chlorosulfoxides as enantioenriched chiral carbenoid sources, see: (c) P. R. Blakemore and M. S. Burge, J. Am. Chem. Soc., 2007, 129, 3068-3069; (d) C. R. Emerson, L. N. Zakharov and P. R. Blakemore, Org. Lett., 2011, 13, 1318-1321; (e) X. Sun and P. R. Blakemore, Org. Lett., 2013, 15, 4500-4503.

53 D. Hoppe, F. Hintze and P. Tebben, Angew. Chem., Int. Ed. Engl., 1990, 12, 1422-1424.

54 M. J. Dearden, C. R. Firkin, J.-P. R. Hermet and P. O'Brien, J. Am. Chem. Soc., 2002, 124, 11870-11871.

55 (a) G. Dutheuil, M. P. Webster, P. A. Worthington and V. K. Aggarwal, Angew. Chem., Int. Ed., 2009, 48, 6317-6319; (b) C. A. Brown and V. K. Aggarwal, Chem. - Eur. J., 2015, 21, 13900-13903; (c) A. Millán, J. R. Smith, J. L. Y. Chen and V. K. Aggarwal, Angew. Chem., Int. Ed., 2016, 55, 2498-2502; (d) A. Noble, S. Roesner and V. K. Aggarwal, Angew. Chem., Int. Ed., 2016, 55, 15920-15924; (e) A. Varela, L. K. B. Garve, D. Leonori and V. K. Aggarwal, Angew. Chem., Int. Ed., 2017, 56, 2127-2131.

56 D. J. Blair, C. J. Fletcher, K. M. P. Wheelhouse and V. K. Aggarwal, Angew. Chem., Int. Ed., 2014, 53, 5552-5555.

57 C. G. Watson, A. Balanta, T. G. Elford, S. Essafi, J. N. Harvey and V. K. Aggarwal, J. Am. Chem. Soc., 2014, 136, 17370-17373.

58 M. Burns, S. Essafi, J. R. Bame, S. P. Bull, M. P. Webster, S. Balieu, J. W. Dale, C. P. Butts, J. N. Harvey and V. K. Aggarwal, Nature, 2014, 513, 183-188.

59 S. Balieu, G. E. Hallett, M. Burns, T. Bootwicha, J. Studley and V. K. Aggarwal, J. Am. Chem. Soc., 2015, 137, 4398-4403.

60 G. Zweifel, H. Arzoumanian and C. C. Whitney, J. Am. Chem. Soc., 1967, 89, 3652-3653.

61 D. A. Evans, T. C. Crawford, R. C. Thomas and J. A. Walker, J. Org. Chem., 1976, 41, 3947-3953.

62 C. J. Fletcher, D. J. Blair, K. M. P. Wheelhouse and V. K. Aggarwal, Tetrahedron, 2012, 68, 7598-7604.

63 For an example of $E$ olefin synthesis by syn elimination of a borane, see: G. Zweifel, R. P. Fisher, J. T. Snow and C. C. Whitney, J. Am. Chem. Soc., 1972, 94, 6560-6561.

64 R. J. Armstrong, C. García-Ruiz, E. L. Myers and V. K. Aggarwal, Angew. Chem., Int. Ed., 2017, 56, 786-790.

65 For an alternative recent approach employing an enantioenriched boronic ester to provide access to either alkene isomer, see: $\mathrm{Z}$. Wu, X. Sun, K. Potter, Y. Cao, L. N. Zakharov and P. R. Blakemore, Angew. Chem., Int. Ed., 2016, 55, 12285-12289.

66 H. C. Brown and M. Srebnik, Organometallics, 1987, 6, 629-631.

67 Y. Wang, A. Noble, E. L. Myers and V. K. Aggarwal, Angew. Chem., Int. Ed., 2016, 55, 4270-4274.

68 A. Ros and V. K. Aggarwal, Angew. Chem., Int. Ed., 2009, 48, 6289-6292.

69 (a) C. Zhang and J. Yun, Org. Lett., 2013, 15, 3416-3419 for a comparable example to form lactams from trifluoroborate salts, see: (b) S. Lee, W. M. Lee and J. Yun, Adv. Synth. Catal., 2015, 357, 2219-2222.
70 M. Mohiti, C. Rampalakos, K. Feeney, D. Leonori and V. K. Aggarwal, Chem. Sci., 2014, 5, 602-607.

71 (a) V. F. Slagt, A. H. M. de Vries, J. G. de Vries and R. M. Kellogg, Org. Process Res. Dev., 2010, 14, 30-47; (b) A. Suzuki, Angew. Chem., Int. Ed., 2011, 50, 6722-6737.

72 (a) K. Matos and J. A. Soderquist, J. Org. Chem., 1998, 63, 461-470; (b) R. Jana, T. P. Pathak and M. S. Sigman, Chem. Rev., 2011, 111, 1417-1492.

73 For selected recent reviews, see: (a) D. Leonori and V. K. Aggarwal, Angew. Chem., Int. Ed., 2015, 54, 1082-1096; (b) C.-Y. Wang, J. Derosa and M. R. Biscoe, Chem. Sci., 2015, 6, 5105-5113; (c) A. H. Cherney, N. T. Kadunce and S. E. Reisman, Chem. Rev., 2015, 115, 9587-9652.

74 (a) D. Imao, B. W. Glasspole, V. S. Laberge and C. M. Crudden, J. Am. Chem. Soc., 2009, 131, 5024-5025; see also: (b) C. M. Crudden, C. Ziebenhaus, J. P. G. Rygus, K. Ghozati, P. J. Unsworth, M. Nambo, S. Voth, M. Hutchinson, V. S. Laberge, Y. Maekawa and D. Imao, Nat. Commun., 2016, 7, 11065.

75 J. Uenishi, J. M. Beau, R. W. Armstrong and Y. Kishi, J. Am. Chem. Soc., 1987, 109, 4756-4758.

76 D. C. Matthew, B. W. Glasspole, P. Eisenberger and C. M. Crudden, J. Am. Chem. Soc., 2014, 136, 5828-5831.

77 Y. Lou, P. Cao, T. Jia, Y. Zhang, M. Wang and J. Liao, Angew. Chem., Int. Ed., 2015, 54, 12134-12138.

78 L. Li, S. Zhao, A. Joshi-Pangu, M. Diane and M. R. Biscoe, J. Am. Chem. Soc., 2014, 136, 14027-14030.

79 M. R. Biscoe, B. P. Fors and S. L. Buchwald, J. Am. Chem. Soc., 2008, 130, 6686-6687.

80 S. D. Dreher, P. G. Dormer, D. L. Sandrock and G. A. Molander, J. Am. Chem. Soc., 2008, 130, 9257-9259.

81 (a) A. Bonet, M. Odachowski, D. Leonori, S. Essafi and V. K. Aggarwal, Nat. Chem., 2014, 6, 584-589; (b) M. Odachowski, A. Bonet, S. Essafi, P. Conti-Ramsden, J. N. Harvey, D. Leonori and V. K. Aggarwal, J. Am. Chem. Soc., 2016, 138, 9521-9532 for examples with boranes, see: $(c)$ E. R. Marinelli and A. B. Levy, Tetrahedron Lett., 1979, 25, 2313-2316; (d) I. Akimoto and A. Suzuki, Synthesis, 1979, 146-147; (e) M. Ishikura, W. Ida and K. Yanada, Tetrahedron, 2006, 62, 1015-1024.

82 J. Llaveria, D. Leonori and V. K. Aggarwal, J. Am. Chem. Soc., 2015, 137, 10958-10961.

83 Y. Wang, A. Noble, C. Sandford and V. K. Aggarwal, Angew. Chem., Int. Ed., 2017, 56, 1810-1814.

84 (a) K. Kubota, E. Yamamoto and H. Ito, J. Am. Chem. Soc., 2015, 137, 420-424; (b) N. Hu, G. Zhao, Y. Zhang, X. Liu, G. Li and W. Tang, J. Am. Chem. Soc., 2015, 137, 6746-6749; (c) V. M. Shoba, N. C. Thacker, A. J. Bochat and J. M. Takacs, Angew. Chem., Int. Ed., 2016, 55, 1465-1469; (d) Y. Xi and J. F. Hartwig, J. Am. Chem. Soc., 2016, 138, 6703-6706; (e) G. Casoni, E. L. Myers and V. K. Aggarwal, Synthesis, 2016, 3241-3253; $(f)$ J. Schmidt, J. Choi, A. Tianxiang Liu, M. Slusarczyk and G. C. Fu, Science, 2016, 354, 1265-1269; $(g)$ D. J. Blair, D. Tanini, J. M. Bateman, H. K. Scott, E. L. Myers and V. K. Aggarwal, Chem. Sci., 2017, 8, 2898-2903. 